TRANSACTIONS OF THE

AMERICAN MATHEMATICAL SOCIETY

Volume 355, Number 4, Pages 1365-1399

S 0002-9947(02)03210-5

Article electronically published on December 2, 2002

\title{
EXISTENCE AND UNIQUENESS \\ FOR A SEMILINEAR ELLIPTIC PROBLEM \\ ON LIPSCHITZ DOMAINS \\ IN RIEMANNIAN MANIFOLDS II
}

\author{
MARTIN DINDOŠ
}

\begin{abstract}
Extending our recent work for the semilinear elliptic equation on Lipschitz domains, we study a general second-order Dirichlet problem $L u-$ $F(x, u)=0$ in $\Omega$. We improve our previous results by studying more general nonlinear terms $F(x, u)$ with polynomial (and in some cases exponential) growth in the variable $u$. We also study the case of nonnegative solutions.
\end{abstract}

\section{INTRODUCTION}

This is a continuation of our paper "Existence and uniqueness for a semilinear elliptic problem on Lipschitz domains in Riemannian manifolds" 3. In that paper we initiated a program aimed at extending results for $L^{p}$ Dirichlet and Neumann boundary problems for linear second-order elliptic equations on Lipschitz domains to the class of semilinear elliptic problems.

We recall the general setting of [3], which will also be in effect in this paper. Let $M$ be a smooth, compact Riemannian manifold of real dimension $\operatorname{dim} M=n \geq 3$, with a Riemannian metric tensor, which is assumed to be Lipschitz. Let $\Omega \subset M$ be a connected Lipschitz domain in $M$. The equation we consider can be written as

$$
L u-F(x, u)=0 \text { in } \Omega,\left.\quad u\right|_{\partial \Omega}=g \in L^{p}(\partial \Omega),
$$

where $L$ is a second-order strongly elliptic, (formally) selfadjoint, negative definite differential operator that can be locally written on a coordinate chart $U \subset M$ as

$$
\left.L\right|_{U}=\sum_{j, k} \frac{\partial}{\partial x_{j}} a_{j k} \frac{\partial}{\partial x_{k}}+\sum_{j} b_{j} \frac{\partial}{\partial x_{j}}+c,
$$

with coefficients

$$
a_{j k} \in \operatorname{Lip}, b_{j} \in H^{1, r}, c \in L^{r / 2},
$$

for some $r>n$. (Actually in [3] it was assumed that $L=\Delta$ is the Laplace-Beltrami operator; we will extend the results of [3] for the more general operator $L$.)

If the function $F$ is differentiable in the variable $u$, we can rewrite the equation in a more convenient form:

$$
L u-a(x, u) u=f \text { in } \Omega,\left.\quad u\right|_{\partial \Omega}=g \in L^{p}(\partial \Omega),
$$

Received by the editors September 11, 2001 and, in revised form, July 24, 2002.

2000 Mathematics Subject Classification. Primary 35J65, 35B65; Secondary 46E35, 42B20.

Key words and phrases. Nonlinear equations, semilinear elliptic problems, Dirichlet boundary problems, Lipschitz domains, Riemannian manifolds. 
where $f(x)=F(x, 0)$ and

$$
a(x, u)=\int_{0}^{1} \frac{\partial}{\partial u} F(x, t u) d t .
$$

The main idea in [3], which allowed us to solve the equation (1.4), was to use results for the linear equation and a fixed point theorem on a new class of spaces $\mathcal{D}^{s, p}$. These spaces were introduced in a simplified version in [4]; the full definition with a list of properties was given in [3]. (Additional properties of the spaces $\mathcal{D}^{s, p}$ are studied in section 2, below.)

However, in the paper [3] we placed one strong restriction on the function $a$ in equation (1.4). Namely, for $p<\infty$ we assumed that

$$
a(x, u) \in L^{\infty}(\Omega \times \mathbb{R}) .
$$

The main goal of this paper is to replace the condition (1.6) by a more general condition that allows polynomial growth in the variable $u$. Let us also mention that in [3] we also studied the Neumann boundary problem, which we will not consider here.

For references to the linear theory, see papers by Verchota [24, Fabes, Jodeit and Rivère [9], Dahlberg and Kenig [7] and others. These papers apply the method of layer potentials on Lipschitz domains in the flat space $\mathbb{R}^{n}$. Recently, a new development for the linear problem was carried out by Mitrea and Taylor (see [19], [20], [21], [22]), who brought the subject to the variable coefficient setting on Lipschitz domains in compact Riemannian manifolds.

Before we give a list of results, let us briefly mention results by other authors for the equation (1.1) and related problems. The two-dimensional planar case for $L=\Delta$ was recently considered by Isakov and Nachman [12]. Their approach is purely variational with use of the maximum principle. The result on the Dirichlet problem obtained by them requires boundary data bounded and having at least half a derivative, i.e., $H^{1 / 2,2}(\partial \Omega)$. Here $H^{s, p}$ stands for a standard Sobolev space of $L^{p}$ integrable functions, $1 \leq p \leq \infty$, with $s$ derivatives, $s \in \mathbb{R}$. For the nonlinear equation (1.1) in dimension greater than two, there are some results discussed in the book by Gilbarg and Trudinger [11]. Their assumption on the boundary data is $H^{(2 p-1) / p, p}$ with $p>n$. They also require the boundary to be at least of the class $C^{2, \alpha}$, which naturally excludes Lipschitz domains. Positive solutions were investigated in the papers by Chen, Williams and Zhao [2] and also by Jin [13]. There is a slight overlap with their work, in particular Theorem 5.1. Finally, our recent joint work with Marius Mitrea [5] considers the problem presented here in Sobolev-Besov spaces.

To end this introduction let us briefly give an overview of the main results of this paper. These results will be established in sections 4-6, after the necessary tools are developed in sections 2 and 3.

For $2-\varepsilon<p<\infty$ we will show that equation (1.4) has a solution $u \in \mathcal{D}^{0, p}$, provided $a(x, u)$ is a Carathéodory function such that

$$
0 \leq a(x, u) \leq k_{1}(x)+k_{2}(x)|u|^{m},
$$

for some $k_{1}, k_{2} \geq 0, k_{1} \in L^{s}(\Omega), k_{2} \in L^{t}(\Omega)(s, t>n / 2)$ and

$$
0 \leq m<p \frac{2-n / t}{n-1}
$$


It is also assumed that $f \in \mathcal{L}_{r}^{q}(\Omega)$, for some $r, q$ (see Definition 2.2 and Theorem 4.3). If, in addition, $F(x, u)=a(x, u) u-f$ satisfies

$$
0 \leq \frac{\partial}{\partial u} F(x, u) \leq k_{1}(x)+k_{2}(x)|u|^{m},
$$

with $k_{1}, k_{2}, m$ as before, we also have uniqueness. If $p=\infty$, instead of (1.7) it suffices to assume

$$
\text { for any } M>0: \quad \sup _{u \in[-M, M]} a(x, u) \in L^{s}(\Omega) \text { for some } s>n / 2, \quad a(x, u) \geq 0 .
$$

For uniqueness one has to require

$$
\text { for any } M>0: \sup _{u \in[-M, M]} \frac{\partial}{\partial u} F(x, u) \in L^{s}(\Omega) \text { for some } s>n / 2, \frac{\partial}{\partial u} F(x, u) \geq 0 .
$$

If the function $a$ satisfies

$$
0 \leq a(x, u) \leq k(x) e^{A|u|}, \quad \text { for some } A>0 \text { and } k \in L^{s}(\Omega)(s>n / 2),
$$

then there exists $C>0$ such that the equation (1.4) is solvable for any $\left.u\right|_{\partial \Omega}=g$ with $g=g_{1}+g_{2}$, where $g_{1} \in L^{\infty}(\partial \Omega)$ and $\left\|g_{2}\right\|_{\mathrm{BMO}(\partial \Omega)}<C$. If (1.12) holds for any $A>0$, we can take any $g \in \mathrm{BMO}(\partial \Omega)$. Uniqueness requires a condition similar to (1.12) for the function $\frac{\partial}{\partial u} F(x, u)$.

Finally, we have a stronger result for nonnegative solutions. For any $2-\varepsilon<p \leq$ $\infty$, if (1.11) holds and $g \in L^{p}(\partial \Omega), g \geq 0$, and $f \in \mathcal{L}_{r}^{q}(\partial \Omega), f \leq 0$, then there exists a unique nonnegative solution $u \in \mathcal{D}^{0, p}$ to (1.4).

\section{The OpERATOR $L^{-1}$ ACTING ON $L^{r}(\Omega) \times \mathcal{D}^{s, p}$}

In section 3 of the paper 3 we considered actions of the inverse $L^{-1}$ of the linear operator

$$
L=\Delta-V
$$

on the Banach space $\mathcal{D}^{s, p}$. Here $\Delta$ is the Laplace-Beltrami operator on a Riemannian manifold $M$, and $V \in L^{\infty}(M), V \geq 0$, is a given function.

The goal of this part is to extend our understanding of the actions of $L^{-1}$ in case $L$ is a more general second-order elliptic operator, as well as the case when $L^{-1}$ acts on functions that are products of functions from $L^{r}(\Omega)$ and $\mathcal{D}^{s, p}$ for some $1 \leq p \leq \infty$ and $r>n / 2$. Our main result (Theorem 2.3) shows that $L^{-1}$ is a smoothing operator, i.e., there is a gain of regularity. Before we state this result we introduce two definitions.

First, let us recall briefly the definition of the space $\mathcal{D}^{s, p}$ from [3. Throughout the paper $\Omega \subset M$ will always be an open, connected domain in $M$ with Lipschitz boundary. Let $\{\gamma(x) ; x \in \partial \Omega\}$ be a collection of nontangential approach regions (as in [3]) such that $\{\gamma(x) ; x \in \partial \Omega\}$ covers $\Omega$ and for any point $y \in \Omega$ the $(n-1)$ dimensional surface measure of the set $\{x \in \partial \Omega ; y \in \gamma(x)\}$ is proportional to $\operatorname{dist}(y, \partial \Omega)^{n-1}$. This guarantees that the function $\mathcal{M}^{s} f$ defined below gives us good information about the $C_{\mathrm{loc}}^{s}(\Omega)$ norm of $f$. 
Definition 2.1. For any $f: \Omega \rightarrow \mathbb{R}, x \in \partial \Omega$ and $s \geq 0$ we consider the number $(2.2)$

$$
\mathcal{M}^{s} f(x)= \begin{cases}\sum_{|\alpha| \leq s} \sup _{z \in \gamma(x)}\left|D^{\alpha} u\right|, & \text { if } s \in \mathbb{Z}, \\ \sum_{|\alpha| \leq k} \sup _{z \in \gamma(x)}\left|D^{\alpha} u\right|+\sum_{|\alpha|=k} \sup _{z, z^{\prime} \in \gamma(x)} \frac{\left|D^{\alpha} u(z)-D^{\alpha} u\left(z^{\prime}\right)\right|}{\operatorname{dist}\left(z, z^{\prime}\right)^{s-k}}, & \text { otherwise. }\end{cases}
$$

Here $k$ is the integer part of $s$, and $\alpha=\left(\alpha_{1}, \alpha_{2}, \ldots, \alpha_{n}\right)$ is a multi-index $(|\alpha|=$ $\left.\sum \alpha_{i}\right)$. We allow $\mathcal{M}^{s} f(x)$ to be infinite for some $x \in \partial \Omega$.

Let $s \geq 0$ and $1 \leq p \leq \infty$. Consider

$$
\mathcal{D}^{s, p}=\left\{f: \Omega \rightarrow \mathbb{R} ; \mathcal{M}^{s} f \in L^{p}(\partial \Omega)\right\},
$$

where $L^{p}(\partial \Omega)$ is the space of $L^{p}$-integrable functions on $\partial \Omega$ with respect to its surface measure. Then $\mathcal{D}^{s, p}$ equipped with the norm

$$
\|f\|_{\mathcal{D}^{s, p}}=\left\|\mathcal{M}^{s} f\right\|_{L^{p}(\partial \Omega)}
$$

is a Banach space.

Remark. As shown in [3], $\mathcal{D}^{s, p}$ does not depend on the choice of the nontangential regions $\{\gamma(x) ; x \in \partial \Omega\}$, i.e., the norms given by (2.4) for two different choices of the nontangential regions are equivalent.

Definition 2.2. Assume that $n / 2<r \leq \infty$ and $1 \leq p \leq \infty$. We say that an integrable function $f: \Omega \rightarrow \mathbb{R}$ belongs to the space $\mathcal{L}_{r}^{p}(\Omega)$ if there are functions $g_{j} \in \mathcal{D}^{0, p}$ and $h_{j} \in L^{r}(\Omega), j=1,2,3, \ldots$, such that $f=\sum_{j} g_{j} h_{j}$ almost everywhere in $\Omega$ and

$$
\sum_{j}\left\|g_{j}\right\|_{\mathcal{D}^{0, p}}\left\|h_{j}\right\|_{L^{r}(\Omega)}<\infty
$$

Moreover, there is a norm on $\mathcal{L}_{r}^{p}(\Omega)$ defined by

$$
\|f\|_{\mathcal{L}_{r}^{p}(\Omega)}:=\|f\|_{L^{1}(\Omega)}+\inf \left\{\sum_{j}\left\|g_{j}\right\|_{\mathcal{D}^{0, p}}\left\|h_{j}\right\|_{L^{r}(\Omega)} ; f=\sum_{j} g_{j} h_{j} \text { a.e. on } \Omega\right\}
$$

that makes $\mathcal{L}_{r}^{p}(\Omega)$ a Banach space.

Remark. We omit the proof of the fact that $\mathcal{L}_{r}^{p}(\Omega)$ is a Banach space, since it is a simple summation argument. It is also helpful to observe that

$$
p>r(n-1) /(r-1) n \Rightarrow\|g h\|_{L^{1}(\Omega)} \leq C\|g\|_{\mathcal{D}^{0, p}}\|h\|_{L^{r}(\Omega)} .
$$

Thus, for $p$ large, $\|f\|_{L^{1}(\Omega)}$ may be omitted from (2.6). Also, $\mathcal{L}_{\infty}^{p}(\Omega)=\mathcal{D}^{0, p}$.

Now we are ready to state the main theorem of this section, about smoothing properties of $L^{-1}$.

Theorem 2.3. Let $L^{-1}$ be an operator acting on functions on an $n$-dimensional compact Riemannian manifold $M$ that can be formally written as

$$
L^{-1} f(x)=\int_{M} E(x, y) f(y) d \operatorname{Vol}(y), \quad x \in M,
$$


where for some $C>0$ and $\beta>0$ the Schwartz kernel $E(x, y)$ satisfies

$$
\begin{aligned}
|E(x, y)| & \leq C d(x, y)^{-(n-2)}, \\
\nabla_{x} E(x, y) \mid & \leq \mid C d(x, y)^{-(n-1)},
\end{aligned}
$$

$$
\begin{aligned}
& \frac{\left|\nabla_{x} E(x, y)-\nabla_{x} E\left(x_{0}, y\right)\right|}{d\left(x, x_{0}\right)^{\beta}} \leq C d\left(x_{0}, y\right)^{-(n-1+\beta)}, \quad \text { for } d\left(x, x_{0}\right) \leq \frac{1}{4} d\left(x_{0}, y\right), \\
& \frac{\left|\nabla_{x} E(x, y)-\nabla_{x} E\left(x_{0}, y\right)\right|}{d\left(x, x_{0}\right)^{\beta}} \leq C\left(d\left(x_{0}, y\right)+d(x, y)\right)^{-(n-1+\beta)}, \\
& \quad \text { for } d\left(x, x_{0}\right)>\frac{1}{4} d\left(x_{0}, y\right) .
\end{aligned}
$$

Here $d(x, y)$ denotes the geodesic distance on $M$ between points $x$ and $y$.

Given any $f \in \mathcal{L}_{r}^{p}(\Omega)$, let $\widetilde{f}$ be the extension of $f$ onto $M$ defined by $\widetilde{f}(x)=0$, for all $x \in M \backslash \bar{\Omega}$. Then the following results hold:

(a) If $r>n$ and $1 \leq p \leq \infty$, then the map

$$
L^{-1}: \mathcal{L}_{r}^{p}(\Omega) \rightarrow \mathcal{D}^{1+\varepsilon, q}
$$

is well defined, continuous and compact for any $1 \leq q \leq \infty$ such that

$$
\frac{1}{q}>\frac{1}{p}+\frac{1}{n-1}\left(\frac{n}{r}-1\right)
$$

and $\varepsilon=\varepsilon(p, q, r)>0$ small. Hence, there is a constant $C=C(p, q, r)>0$ such that

$$
\left\|L^{-1}(\widetilde{f})\right\|_{\mathcal{D}^{1+\varepsilon, q}} \leq C\|f\|_{\mathcal{L}_{r}^{p}(\Omega)} .
$$

(b) If $r>n / 2$ and $1 \leq p \leq \infty$, then the map

$$
L^{-1}: \mathcal{L}_{r}^{p}(\Omega) \rightarrow \mathcal{D}^{\varepsilon, q}
$$

is well defined, continuous and compact for any $1 \leq q \leq \infty$ such that

$$
\frac{1}{q}>\frac{1}{p}+\frac{2}{n-1}\left(\frac{n}{2 r}-1\right),
$$

and $\varepsilon=\varepsilon(p, q, r)>0$ small. Hence, there is a constant $C=C(p, q, r)>0$ such that

$$
\left\|L^{-1}(\widetilde{f})\right\|_{\mathcal{D}^{\varepsilon, q}} \leq C\|f\|_{\mathcal{L}_{r}^{p}(\Omega)} .
$$

We will prove Theorem 2.3 in a series of smaller lemmas and propositions. There are many examples of operators satisfying (2.9). Consider the following setting from [19.

Let $L$ be a general second-order, strongly elliptic, formally selfadjoint operator acting on sections of a vector bundle $\mathcal{E}$ over a compact Riemannian manifold $M$ of dimension $n \geq 3$. Assume that the Hermitian structure on $\mathcal{E}$ has $C^{1}$ coefficients, that the metric tensor $g$ on $M$ is $C^{1}$, i.e.,

$$
g_{i j} \in C^{1}(M)
$$

and that, in local coordinates $U \subset M$ over which $\mathcal{E}$ trivializes,

$$
\left.L\right|_{U}=\sum_{j, k} \sum_{\alpha, \beta} \frac{\partial}{\partial x_{j}} a_{j k}^{\alpha \beta} \frac{\partial}{\partial x_{k}}+\sum_{j} \sum_{\alpha \beta} b_{j}^{\alpha \beta} \frac{\partial}{\partial x_{j}}+\sum_{\alpha \beta} c^{\alpha \beta}
$$


where

$$
a_{j k}^{\alpha \beta} \in C^{1}, b_{j}^{\alpha \beta} \in L^{\infty}, c^{\alpha \beta} \in L^{r}, r>n / 2 .
$$

Let us note that the formal selfadjointness hypothesis can be relaxed to

$$
\operatorname{degree}\left(L-L^{*}\right) \leq 1 \text {. }
$$

Since we want to consider boundary value problems on $\Omega \subset M$, the operator $L$ could be conveniently modified on $M \backslash \bar{\Omega}$ to satisfy the required hypotheses.

A basic example of an operator $L$ satisfying these hypotheses is provided by the Hodge Laplacian

$$
\Delta=-\delta d-d \delta
$$

acting on sections of the $\ell$-th exterior power of the tangent bundle of a Riemannian manifold $M$ satisfying (2.16) in the absence of global $\ell$-harmonic fields, $1 \leq \ell \leq$ $n-1$, assuming also that $g_{i j} \in H^{2, r}$ for some $r>n$.

In the scalar case $(\mathcal{E}=\mathbb{R})$, which is our primary concern, (2.20) takes the standard form in local coordinates:

$$
\Delta u=g^{-1 / 2} \partial_{j}\left(g^{j k} g^{1 / 2} \partial_{k} u\right)
$$

We use the summation convention, take $\left(g^{j k}\right)$ to be the inverse matrix to $\left(g_{j k}\right)$, and set $g=\operatorname{det}\left(g_{j k}\right)$. As shown in [22], we can relax (2.16) and assume $g \in C^{\alpha}$ for some $\alpha>0$ in this case.

The estimates $(2.9)$ on the kernel $E(x, y)$ for the operator $L$ of the form $(2.17)$ we are seeking are obtained in 19 . Proposition 2.5 together with other considerations in section 2 of 19 give us that (2.9) holds, provided the metric tensor $g$ is $C^{1}$, both $L$ and $L^{*}$, locally given by $(2.17)$, satisfy $(2.18)$, and $L$ is invertible as a map from $H^{1,2}(M, \mathcal{E})$ onto $H^{-1,2}(M, \mathcal{E})$.

Obviously, at least in the scalar case, the assumptions (2.16) and (2.18) could be further relaxed following [21] and [22]. We are not going to try to achieve the lowest possible regularity of the coefficients, as was presented in 22] for the Laplace operator, since this would add additional difficulties to our exposition. We note, however, that instead of assuming (2.16) it suffices to have a Lipschitz metric tensor, as was shown in [21].

Now we begin a sequence of small reductions which simplify our problem. First, as follows from the definition of the space $\mathcal{L}_{r}^{p}(\Omega)$, it is enough to prove the estimates (2.12) and (2.15) for functions in $\mathcal{L}_{r}^{p}(\Omega)$ of the form $f g$, where $f \in L^{r}(\Omega)$ and $g \in \mathcal{D}^{0, p}$.

Secondly, as our steps closely resemble section 3 of [3], we maintain brevity by pointing out only the most important differences. We start with an analogue of Lemma 3.1 of [3]. From now on we work mainly in local coordinates; hence in (2.9), we can replace the geodesic distance $d(x, y)$ between two points by $|x-y|$, the Euclidean distance between these points in the local coordinates.

Lemma 2.4. Assume that (2.9) holds. Let $x \in M$ be an arbitrary point and $r>0$. Consider a geodesic ball $B_{r}(x)$ of radius $r$ around $x$, and assume that $g \in L^{\infty}(M)$ is a given function with support in $B_{r}(x)$ and bounded in absolute value by one on $M$. Let $f$ be a function in $L^{p}(M)$ and let $u$ solve the equation

$$
L u=f g \quad \text { in } M, \quad \text { i.e., } \quad u=L^{-1}(f g) .
$$


If $p>n / 2$, then there are an $\varepsilon>0$ and a constant $C=C(p)$ such that for any $y, y^{\prime} \in M$,

$$
\begin{gathered}
|u(y)| \leq \mid C \frac{r^{n(p-1) / p}}{(r+|x-y|)^{n-2}}\|f\|_{L^{p}(M)}, \\
\frac{\left|u(y)-u\left(y^{\prime}\right)\right|}{\left|y-y^{\prime}\right|^{\varepsilon}} \leq C \frac{r^{n(p-1) / p}}{\left(r+\min \left\{|x-y|,\left|x-y^{\prime}\right|\right\}\right)^{n-2+\varepsilon}}\|f\|_{L^{p}(M)} .
\end{gathered}
$$

Also, for such $y, y^{\prime}$, if $d_{y}=\operatorname{dist}\left(y, B_{r}(x)\right)$ and $d_{y^{\prime}}=\operatorname{dist}\left(y^{\prime}, B_{r}(x)\right)$, then

$$
|u(y)| \leq \frac{C}{d_{y}^{n-2}}\|f g\|_{L^{1}(M)} \quad \text { and } \quad \frac{\left|u(y)-u\left(y^{\prime}\right)\right|}{\left|y-y^{\prime}\right|^{\varepsilon}} \leq C \frac{\|f g\|_{L^{1}(M)}}{\min \left\{d_{y}, d_{y^{\prime}}\right\}^{n-2+\varepsilon}} .
$$

If $p>n$, then there are an $\varepsilon>0$ and a constant $C=C(p)$ such that for any $y, y^{\prime} \in M$

$$
\begin{gathered}
|\nabla u(y)| \leq \mid C \frac{r^{n(p-1) / p}}{(r+|x-y|)^{n-1}}\|f\|_{L^{p}(M)}, \\
\frac{\left|\nabla u(y)-\nabla u\left(y^{\prime}\right)\right|}{\left|y-y^{\prime}\right|^{\varepsilon}} \leq C \frac{r^{n(p-1) / p}}{\left(r+\min \left\{|x-y|,\left|x-y^{\prime}\right|\right\}\right)^{n-1+\varepsilon}}\|f\|_{L^{p}(M)} .
\end{gathered}
$$

Proof. We show only (2.23), since (2.25) is very similar. Using the Hölder inequality, we can estimate

$$
|u(y)| \leq \int_{B_{r}}|E(y, z)||f(z)| d \operatorname{Vol}(z) \leq v(y)^{(p-1) / p}\|f\|_{L^{p}(M)},
$$

where

$$
v(y)=\int_{B_{r}}|E(y, z)|^{p /(p-1)} d \operatorname{Vol}(z)
$$

Similarly,

$$
\frac{\left|u(y)-u\left(y^{\prime}\right)\right|}{\left|y-y^{\prime}\right|^{\varepsilon}} \leq w\left(y, y^{\prime}\right)^{(p-1) / p}\|f\|_{L^{p}(M)},
$$

where

$$
w\left(y, y^{\prime}\right)=\int_{B_{r}}\left|\frac{E(y, z)-E\left(y^{\prime}, z\right)}{\left|z-z^{\prime}\right|^{\varepsilon}}\right|^{p /(p-1)} d \operatorname{Vol}(z) .
$$

The integrals (2.27) and (2.29) are very similar to the integrals estimated in the proof of Lemma 3.1 of [3]. For this reason we consider closely only (2.27).

First, we take $y \in B_{2 r}(x)$. We estimate $v(y)$ using (2.9). Assume for simplicity that $r>0$ is small enough so that we can consider just one geodesic coordinate chart centered at $x$ that contains the ball $B_{2 r}(x)$. In this chart we can also assume that $x$ is at the origin. We integrate over $(n-1)$-dimensional shells $S_{\rho}=\partial B_{\rho}(y)$ centered at $y$. A simple estimate using (2.9) gives

$$
\begin{aligned}
v(y) & \leq C \int_{0}^{3 r} \int_{S_{\rho}}\left(\frac{1}{|z-y|^{n-2}}\right)^{p /(p-1)} d \sigma(z) d \rho \\
& =C \int_{0}^{3 r} \int_{S_{\rho}} \frac{1}{|z-y|^{(n-2) p /(p-1)}} d \sigma(z) d \rho .
\end{aligned}
$$


Since the surface area of $S_{\rho}$ is of the order of $\rho^{n-1}$ from (2.30) we get for $p>n / 2$ that $n-1-(n-2) p /(p-1)>-1$, i.e., the function inside the following integral is integrable:

$$
v(y) \leq C \int_{0}^{3 r} \rho^{n-1-(n-2) p /(p-1)} d \rho \leq C r^{n-(n-2) p /(p-1)} .
$$

(2.31) together with (2.26) yields

$$
|u(y)| \leq C r^{2-n / p}\|f\|_{L^{p}(M)} .
$$

By possibly enlarging the constant $C$ in $(2.23)$ we can see that (2.32) and the first line of (2.23) are equivalent for $y \in B_{2 r}(x)$.

Now we consider $y$ outside the ball $B_{2 r}(x)$. Denote by $d_{y}$ the distance between $y$ and $B_{r}(x)$ and by $d$ the distance between $x$ and $y$. Clearly for $y \notin B_{2 r}(x)$ we have $d \approx d_{y}$. Also, for $z \in B_{r}(x)$ we have $|E(y, z)| \leq C d_{y}^{-(n-2)} \approx C d^{-(n-2)}$. The volume of $B_{r}(x)$ is of the order $r^{n}$, which gives

$$
|u(y)| \leq \int_{B_{r}(x)}\left|E(y, z)\left\|f(z) \mid d \operatorname{Vol}(z) \leq \frac{C}{d^{n-2}}\right\| f\left\|_{L^{1}\left(B_{r}\right)} \leq C \frac{r^{n(p-1) / p}}{d^{n-2}}\right\| f \|_{L^{p}(M)} .\right.
$$

(2.33) is equivalent to the first line of (2.23) for $y \notin B_{2 r}(x)$. To see (2.24) one should make an estimate very similar to (2.33). For $d_{y}=\operatorname{dist}\left(y, B_{r}(x)\right)$ we get that $|E(y, z)| \leq C d_{y}^{-(n-2)}$ for $z \in B_{r}(x)$. Hence

$$
|u(y)| \leq \int_{B_{r}(x)}\left|E(y, z)\left\|f(z) g(z) \mid d \operatorname{Vol}(z) \leq \frac{C}{d_{y}^{n-2}}\right\| f g\left\|_{L^{1}\left(B_{r}\right)}=\frac{C}{d_{y}^{n-2}}\right\| f g \|_{L^{1}(M)} .\right.
$$

Next we establish an analogue of Proposition 3.2 from [3].

Proposition 2.5. Assume that $r>0$ is small. Let $g \in L^{\infty}(M)$ be a function on $M$ bounded in absolute value by one with support in $B_{r}(x) \cap \Omega$, where $x$ is a point from the boundary $\partial \Omega$, and let $f$ be any function from $L^{p}(M)$. Denote by $u$ the solution to the equation $L u=f g$ in $M$, i.e., $u=L^{-1}(f g)$.

(a) If $p>n$, then there exists $\varepsilon=\varepsilon(p)>0$ such that

$$
\left\|\mathcal{M}^{1+\varepsilon} u\right\|_{L^{q}(\partial \Omega)} \leq C r^{n-1}\|f\|_{L^{p}(M)}, \quad \text { for } 1 \leq q<1+\varepsilon .
$$

(b) If $p>n / 2$, then the maximal operator $\mathcal{M}^{\varepsilon} u$ can be written as

$$
\mathcal{M}^{\varepsilon} u=v^{1}+v^{2}
$$

where

$$
\left\|v^{1}\right\|_{L^{q}(\partial \Omega)} \leq C r^{n-1}\|f\|_{L^{p}}, \quad \text { for any } 1 \leq q<1+\varepsilon_{0}(p) \text { and any } 0 \leq \varepsilon<\varepsilon_{0}(p),
$$

and

$\left\|v^{2}\right\|_{L^{q}(\partial \Omega)} \leq C\|f g\|_{L^{1}}, \quad$ for any $1 \leq q<(n-1) /(n-2)$ and any $0 \leq \varepsilon<\varepsilon_{1}(p, q)$.

The constants $C$ in (2.35), (2.37), and (2.38) might depend on $p$ and $q$, but not on $r$. 
Proof. We first look at (2.35). Since $r>0$ is small, we can find a small neighborhood $U$ of $x$ such that in this neighborhood there are smooth local coordinates in which

$$
U \cap \Omega=\left\{x=\left(x^{\prime}, x_{n}\right) \in U: x_{n}>\varphi\left(x^{\prime}\right)\right\},
$$

where $\varphi$ is a Lipschitz function with a Lipschitz constant bounded by $L$. Here $L$ does not depend on the chosen point $x \in \partial \Omega$. We will consider nontangential approach regions $\gamma(z)$ to any point $z=\left(z^{\prime}, \varphi\left(z^{\prime}\right)\right) \in \partial \Omega$ such that the vertex of $\gamma(z)$ at $z$ is sharp enough. Namely, we require that any half-ray with vertex at $z$ that lies wholly in $\gamma(z)$ has "steepness" (absolute value of its slope) at least $2 L$. Hence in the local coordinates (2.39) we have

$$
\gamma(z)=\left\{y=\left(y^{\prime}, y_{n}\right) ; y_{n}-z_{n}>2 L\left|y^{\prime}-z^{\prime}\right|\right\},
$$

for any $z=\left(z^{\prime}, z_{n}\right) \in \partial \Omega \cap U$.

From this it follows that there exists a universal constant $k$ (independent of $r$ ) such that we can split points $z \in \partial \Omega$ into two distinct sets. If $z=\left(z^{\prime}, \varphi\left(z^{\prime}\right)\right) \in \partial \Omega$ and $\left|z^{\prime}-x^{\prime}\right| \leq k r$, then $\gamma(z)$ might intersect $B_{r}(x)$. At such a point, by (2.23) and (2.25) we have:

$$
\begin{aligned}
\mathcal{M}^{0} u(z) & \leq C r^{2-n / p}\|f\|_{L^{p}(M)}, \quad \mathcal{M}^{0}(\nabla u)(z) \leq C r^{1-n / p}\|f\|_{L^{p}(M)}, \\
\mathcal{T}^{\varepsilon} u(z) & \stackrel{\text { def }}{=} \sup _{y, y^{\prime} \in \gamma(z)} \frac{\left|\nabla u(y)-\nabla u\left(y^{\prime}\right)\right|}{\left|y-y^{\prime}\right|^{\varepsilon}} \leq C r^{1-n / p-\varepsilon}\|f\|_{L^{p}(M)} .
\end{aligned}
$$

The exact choice of $\varepsilon>0$ in (2.41) will be specified later.

On the other hand, if $\left|z^{\prime}-x^{\prime}\right|>k r$, then the distance between any point $w \in \gamma(z)$ and $x$ is greater than or equal to $\frac{1}{k}\left|z^{\prime}-x^{\prime}\right|$. This means that for such $z$ we get from $(2.23)$ and $(2.25)$

$$
\begin{aligned}
\mathcal{M}^{0} u(z) & \leq C \frac{r^{n(p-1) / p}}{\left(r+k^{-1}\left|z^{\prime}-x^{\prime}\right|\right)^{n-2}}\|f\|_{L^{p}(M)}, \\
\mathcal{M}^{0}(\nabla u)(z) & \leq C \frac{r^{n(p-1) / p}}{\left(r+k^{-1}\left|z^{\prime}-x^{\prime}\right|\right)^{n-1}}\|f\|_{L^{p}(M)}, \\
\mathcal{T}^{\varepsilon} u(z)=\sup _{y, y^{\prime} \in \gamma(z)} \frac{\left|\nabla u(y)-\nabla u\left(y^{\prime}\right)\right|}{\left|y-y^{\prime}\right|^{\varepsilon}} & \leq C \frac{r^{n(p-1) / p}}{\left(r+k^{-1}\left|z^{\prime}-x^{\prime}\right|\right)^{n-1-\varepsilon}}\|f\|_{L^{p}(M)} .
\end{aligned}
$$

Now we can estimate the $L^{q}$ norms of $\mathcal{M}^{0} u, \mathcal{M}^{0}(\nabla u)$ and $\mathcal{T}^{\varepsilon} u$. Taking the sum of these three numbers, we get, on $B_{k r}(x) \cap \partial \Omega$,

$$
\int_{B_{k r}(x) \cap \partial \Omega}\left(\mathcal{M}^{1+\varepsilon} u(y)\right)^{q} d \sigma(y) \leq C r^{q(1-\varepsilon-n / p)+n-1}\|f\|_{L^{p}(M)}^{q} .
$$

Similarly, off $B_{k r}(x) \cap \partial \Omega$ we get

$$
\int_{\partial \Omega \backslash B_{k r}(x)}\left(\mathcal{M}^{1+\varepsilon} u(y)\right)^{q} d \sigma(y) \leq C r^{q(1-\varepsilon-n / p)+n-1}\|f\|_{L^{p}(M)}^{q} .
$$

For details, see [3]. Since $p>n$, we can pick $\varepsilon>0$ small for which $1-\varepsilon-n / p>0$. From this for $q=1$ we get that $q(1-\varepsilon-n / p)+n-1>q(n-1)$. By possibly making $\varepsilon>0$ smaller, we can therefore claim that for any $1 \leq q<1+\varepsilon$ we have $q(1-\varepsilon-n / p)+n-1>q(n-1)$. This means that for $|r|$ bounded we can estimate (2.43) and (2.44) from above by $C r^{q(n-1)}\|f\|_{L^{p}(M)}^{q}$.

Now we put (2.43)-(2.44) together. For $p>n$ and any $1 \leq q<1+\varepsilon$ we get

$$
\left\|\mathcal{M}^{1+\varepsilon} u\right\|_{L^{q}(\partial \Omega)} \leq C\left(r^{n-1+q(1-\varepsilon-n / p)}\right)^{1 / q}\|f\|_{L^{p}(M)} \leq C r^{n-1}\|f\|_{L^{p}(M)} .
$$


This gives (2.35). Next we look at (2.36). On the set $B_{k r}(x)$ we can do the same estimate as before, giving us

$$
\int_{B_{k r}(x) \cap \partial \Omega}\left(\mathcal{M}^{0} u(y)\right)^{q} d \sigma(y) \leq C r^{n+q(2-n / p)-1}\|f\|_{L^{p}(M)}^{q} .
$$

Clearly, for any $p>n / 2$ one can see that for any $q \geq 1$ small enough we have $n+q(2-n / p)-1>q(n-1)$. Hence, if $\mathcal{X}_{B_{k r}(x)}$ is the characteristic function of the set $B_{k r}(x)$, we get that for any $p>n / 2$ there is an $\varepsilon=\varepsilon(p)>0$ such that

$$
\left\|\mathcal{X}_{B_{k r}(x)} \mathcal{M}^{0} u\right\|_{L^{q}(\partial \Omega)} \leq C r^{n-1}\|f\|_{L^{p}}, \quad \text { for any } 1 \leq q<1+\varepsilon .
$$

The problematic part is to estimate the $L^{q}$ norm of $\left(1-\mathcal{X}_{B_{k r}(x)}\right) \mathcal{M}^{0} u$. The estimate $(2.42)$ gives that for any $1 \leq q<(n-1) /(n-2)$ (to keep the integral finite) we have

$$
\left\|\left(1-\mathcal{X}_{B_{k r}(x)}\right) \mathcal{M}^{0} u\right\|_{L^{q}(\partial \Omega)} \leq C r^{n p /(p-1)}\|f\|_{L^{p}} .
$$

At this point, for $n / 2<p<n$ clearly $n p /(p-1)<n-1$; hence we cannot estimate the right-hand side of (2.48) from above by $\mathrm{Cr}^{n-1}\|f\|_{L^{p}}$, which would be desirable.

There is a way around. If we use (2.24) instead of (2.23) to estimate the $L^{q}$ norm of $\left(1-\mathcal{X}_{B_{k r}(x)}\right) \mathcal{M}^{0} u$, we get

$$
\int_{\partial \Omega \backslash B_{k r}(x) \cap \partial \Omega}\left(\mathcal{M}^{0} u(y)\right)^{q} d \sigma(y) \leq C\|f g\|_{L^{1}(M)}^{q} \int_{k r}^{A} \int_{S_{\rho}} \frac{1}{\rho^{q(n-2)}} d \sigma(y) d \rho,
$$

where $S_{\rho}=\partial B_{\rho}(x)$. We also used that, for $y \notin B_{k r}(x), d_{y}=\operatorname{dist}\left(y, B_{r}(x)\right) \approx$ $\operatorname{dist}(y, x)=\rho$. Since the $(n-2)$-dimensional measure of $S_{\rho}$ is of the order $\rho^{n-2}$, we get that for any $1 \leq q<(n-1) /(n-2)$,

$$
\left\|\left(1-\mathcal{X}_{B_{k r}(x)}\right) \mathcal{M}^{0} u\right\|_{L^{q}(\partial \Omega)}^{q} \leq C\|f g\|_{L^{1}(M)}^{q} \int_{0}^{A} \rho^{n-2-q(n-2)} d \rho \leq C(q)\|f g\|_{L^{1}(M)}^{q} ;
$$

hence

$$
\left\|\left(1-\mathcal{X}_{B_{k r}(x)}\right) \mathcal{M}^{0} u\right\|_{L^{q}(\partial \Omega)} \leq C(q)\|f g\|_{L^{1}(M)} .
$$

This establishes (2.36)-(2.38) for $\varepsilon=0$. It is not difficult (using (2.23) and (2.24)) to establish estimates similar to $(2.47)$ and $(2.51)$ for the $L^{q}(\partial \Omega)$ norm of

$$
\mathcal{R}^{\varepsilon} u(x)=\sup _{y, y^{\prime} \in \gamma(x)} \frac{\left|u(y)-u\left(y^{\prime}\right)\right|}{\left|y-y^{\prime}\right|^{\varepsilon}}, \quad \text { where } x \in \partial \Omega .
$$

All the above steps will go through, provided $\varepsilon$ is kept sufficiently close to zero.

The previous results are key to the following proposition.

Proposition 2.6. Assume that the function $g: \Omega \rightarrow \mathbb{R}$ belongs to $\mathcal{D}^{0, p}$ for some $1 \leq p \leq \infty$ and that $f \in L^{r}(\Omega)$. Consider the extension $\widetilde{h}$ of the function $h=f g$ defined as in Theorem 2.3, and denote by $u$ the solution to

$$
L u=\widetilde{h} \quad \text { in } M, \quad \text { i.e., } \quad u=L^{-1}(f g) .
$$

(a) If $r>n$, then there exists $\varepsilon=\varepsilon(r, p)>0$ such that the nontangential maximal function of $\mathcal{M}^{1+\varepsilon} u$ belongs to $L^{p}(\partial \Omega)$, and for some $C=C(r, p)>0$,

$$
\|u\|_{\mathcal{D}^{1+\varepsilon, p}} \leq C\|f\|_{L^{r}(\Omega)}\|g\|_{\mathcal{D}^{0, p}} \approx\|f g\|_{\mathcal{L}_{r}^{p}(\Omega)} .
$$


(b) If $r>n / 2$ and $p>r(n-1) /(r-1) n$, then there exists $\varepsilon=\varepsilon(r, p)>0$ such that $u \in \mathcal{D}^{\varepsilon, p}$ and

$$
\|u\|_{\mathcal{D}^{\varepsilon, p}} \leq C(p, r)\|f\|_{L^{r}(\Omega)}\|g\|_{\mathcal{D}^{0, p}} \approx\|f g\|_{\mathcal{L}_{r}^{p}(\Omega)} .
$$

(c) If $r>n / 2,1 \leq p<(n-1) /(n-2)$ and $f g \in L^{1}(\Omega)$, then there exists $\varepsilon=\varepsilon(p, r)>0$ such that $u \in \mathcal{D}^{\varepsilon, p}$ and

$$
\|u\|_{\mathcal{D}^{\varepsilon, p}} \leq C(p, r)\left(\|f\|_{L^{r}(\Omega)}\|g\|_{\mathcal{D}^{0, p}}+\|f g\|_{L^{1}(\Omega)}\right) \approx\|f g\|_{\mathcal{L}_{r}^{p}(\Omega)} .
$$

Remark. Note that the intervals where (2.55) and (2.56) are valid overlaps; hence the whole interval $1 \leq p \leq \infty$ is covered.

Proof. We will be brief, since the proof follows exactly the steps in our paper [3] that led to the proof of Theorem 3.5 of [3]. The main ingredient is a decomposition of a function $g \in L^{\infty}(\Omega),|g(x)| \leq 1$, with support in the set $\operatorname{Pim}(A) \subset \Omega$. The definition of this set is given in Definition 3.3 of [3]. Any such function can be written as $g=\sum_{i_{1}}^{\infty} g_{i}$, where $\left|g_{i}\right| \leq 1$, supp $g_{i} \subset B\left(x_{i}, \ell_{i}\right)$ for some $x_{i} \in \partial \Omega$, $\ell_{i}=K_{0} / 2^{m}, m=0,1,2, \ldots$, and

$$
\|g\|_{\mathcal{D}^{0,1}} \leq \sigma(A) \approx \sum_{i=1}^{\infty} \ell_{i}^{n-1}
$$

Here $\sigma($.$) denotes the surface measure of the set A \subset \partial \Omega$. Given this, Proposition 2.5 implies that for $u=L^{-1}(\widetilde{f g})$,

$$
\left\|\mathcal{M}^{1+\varepsilon} u\right\|_{L^{q}(\partial \Omega)} \leq C\|f\|_{L^{r}(\Omega)} \sigma(A), \quad \text { for } 1 \leq q<1+\varepsilon .
$$

The second estimate for $u$ (for $r>n$ ) is due to classical elliptic regularity:

$$
f g \in L^{r}(\Omega), r>n \Longrightarrow u \in C^{1+\varepsilon}(\Omega)
$$

i.e.,

$$
\left\|\mathcal{M}^{1+\varepsilon} u\right\|_{L^{\infty}(\partial \Omega)} \leq C\|f\|_{L^{r}(\Omega)} .
$$

The rest goes as in [3] (interpolation of (2.58)-(2.60), additional decomposition and use of the Hölder inequality). Details can be found in the proof of Theorem 3.5 of [3]. This proves part (a).

Parts (b) and (c) are similar. We put $u_{i}=L^{-1}\left(f g_{i}\right)$. Since supp $g_{i} \subset B\left(x_{i}, \ell_{i}\right)$, part (b) of Proposition 2.5 applies, giving us that the maximal operator $\mathcal{M}^{\varepsilon} u_{i}$ can be written as $v_{i}^{1}+v_{i}^{2}$, where

$$
\left\|v_{i}^{1}\right\|_{L^{q}(\partial \Omega)} \leq C \ell_{i}^{n-1}\|f\|_{L^{r}}, \quad \text { for any } 1 \leq q<1+\varepsilon_{0}(p) \text { and any } 0 \leq \varepsilon<\varepsilon_{0}(p),
$$

and

$\left\|v_{i}^{2}\right\|_{L^{q}(\partial \Omega)} \leq C\left\|f g_{i}\right\|_{L^{1}}, \quad$ for any $1 \leq q<(n-1) /(n-2)$ and any $0 \leq \varepsilon<\varepsilon_{1}(p, q)$.

We would like to see whether it is possible to control the $L^{\infty}(\partial \Omega)$ norm of $v^{1}=\sum_{i} v_{i}^{1}$.

Pick any $y \in \partial \Omega$ and $i \in \mathbb{N}$, and consider when $v_{i}^{1}(y)>0$. Clearly this happens only if $y \in B\left(x_{i}, k \ell_{i}\right)$, where $k$ is the same constant as in Proposition 2.5. Moreover, we know that $\ell_{i}=K_{0} / 2^{m}$ for some $m=0,1,2, \ldots$

Hence, if we fix $m$, we claim that the cardinality of the set

$$
\mathcal{I}_{m}(y)=\left\{i \in \mathbb{N} ; y \in B_{k \ell_{i}}\left(x_{i}, k \ell_{i}\right) \text { and } \ell_{i}=C_{0} / 2^{m}\right\}
$$


is finite and bounded from above by some constant $K$ that does not depend on the point $y$, the number $m$ or the set $A$. Seeing this is not difficult. Clearly all points $X_{m}=\left\{x_{i} ; i \in \mathcal{I}_{m}(y)\right\}$ belong to a ball of radius $k C_{0} / 2^{m}$ centered at the point $y$. Also it follows from properties (b) and (c) of the decomposition $C_{1}, C_{2}, \ldots$ that the distance between any two distinct points from $X_{m}$ is at least $C / 2^{m}$, with $C$ depending only on the Lipschitz character of the boundary $\partial \Omega$. From this the existence of the constant $K$ follows by a simple geometric argument.

Now, for each $i \in I_{m}(y)$, according to part b) of Proposition 2.5 we can bound $v_{i}^{1}(y)$ by

$$
v_{i}^{1}(y) \leq C \ell_{i}^{2-\varepsilon-n / r}\|f\|_{L^{r}(M)}=C\left(\frac{K_{0}}{2^{m}}\right)^{2-\varepsilon-n / r}\|f\|_{L^{r}(M)} .
$$

Here $\varepsilon>0$ is small enough so that

$$
\theta=2-\varepsilon-n / r>0 .
$$

It follows that

$$
\begin{aligned}
v^{1}(y)=\sum_{i=1}^{\infty} v_{i}^{1}(y) & \leq K \sum_{m=0}^{\infty} C\left(\frac{K_{0}}{2^{m}}\right)^{\theta}\|f\|_{L^{r}(M)} \\
& \leq C\|f\|_{L^{r}(M)} \sum_{m=0}^{\infty} 2^{-m \theta} \leq C(r)\|f\|_{L^{r}(M)}
\end{aligned}
$$

On the other hand, by (2.61) we get

$$
\left\|v^{1}\right\|_{L^{q}(\partial \Omega)} \leq \sum_{i=1}^{\infty}\left\|v_{i}^{1}\right\|_{L^{q}(\partial \Omega)} \leq C \sum_{i=1}^{\infty} \ell_{i}^{n-1}\|f\|_{L^{r}} \leq C \sigma(A)\|f\|_{L^{r}},
$$

for any $1 \leq q<1+\varepsilon_{0}(r)$.

Interpolating between (2.66) and (2.67), we get that for any $1<q<\infty$ there is a $\delta=\delta(q)>0$ such that

$$
\left\|v^{1}\right\|_{L^{q}(\partial \Omega)} \leq C(r)\|f\|_{L^{r}} \sigma(A)^{1 / q+\delta} .
$$

On the other hand, (2.62) gives us immediately, for $v^{2}=\sum_{i} v_{i}^{2}$,

$$
\left\|v^{2}\right\|_{L^{q}(\partial \Omega)} \leq \sum_{i=1}^{\infty}\left\|v_{i}^{2}\right\|_{L^{q}(\partial \Omega)} \leq \sum_{i=1}^{\infty} C\left\|f g_{i}\right\|_{L^{1}}=C\|f g\|_{L^{1}(M)}
$$

for any $1 \leq q<(n-1) /(n-2)$.

If we look at $(2.69)$ we see that $\|f g\|_{L^{1}} \leq\|f\|_{L^{r}}\|g\|_{L^{r^{\prime}}}$, where $r^{\prime}=r /(r-1)$. Since $r>n / 2$, clearly $1 \leq r^{\prime}<n /(n-2)$. We also know that

$$
\begin{aligned}
\|g\|_{L^{1}(M)} & \leq \sum \operatorname{Vol}\left(B\left(x_{i}, \ell_{i}\right)\right) \leq C \sigma(A)^{n /(n-1)}, \\
\|g\|_{L^{\infty}(M)} & \leq 1 .
\end{aligned}
$$

The last estimate in the first line follows from (3.77) in [3]. Hence

$$
\|g\|_{L^{r^{\prime}(M)}} \leq C \sigma(A)^{n / r^{\prime}(n-1)} .
$$

Notice also that

$$
\frac{n}{r^{\prime}(n-1)}>\frac{n-2}{n-1}
$$


If we put (2.69)-(2.72) together, we get, for any $r^{\prime}(n-1) / n<q<(n-1) /(n-2)$,

$$
\left\|v^{2}\right\|_{L^{q}(\partial \Omega)} \leq C\|f\|_{L^{r}} \sigma(A)^{n / r^{\prime}(n-1)} \leq C\|f\|_{L^{r}} \sigma(A)^{1 / q+\delta},
$$

where

$$
\delta=\frac{n}{r^{\prime}(n-1)}-\frac{1}{q}>0 .
$$

Finally, we put (2.68) and (2.73) together to get

$$
\begin{aligned}
\left\|\mathcal{M}^{\varepsilon} u\right\|_{L^{q}(\partial \Omega)} & =\left\|\mathcal{M}^{\varepsilon}\left(\sum_{i=1}^{\infty} u_{i}\right)\right\|_{L^{q}(\partial \Omega)} \leq\left\|\sum_{i=1}^{\infty} \mathcal{M}^{\varepsilon} u_{i}\right\|_{L^{q}(\partial \Omega)} \\
& \leq\left\|v^{1}\right\|_{L^{q}(\partial \Omega)}+\left\|v^{2}\right\|_{L^{q}(\partial \Omega)} \leq C\|f\|_{L^{r}} \sigma(A)^{1 / q+\delta}
\end{aligned}
$$

for any $r^{\prime}(n-1) / n<q<(n-1) /(n-2)$ and some $\varepsilon=\varepsilon(r, q)>0$. Here $C=C(r, q)>0$. On the other hand, since $f g \in L^{r}(M)$, for $r>n / 2$ it follows that $u=L^{-1}(f g) \in C^{\varepsilon}(M)$, and hence we have

$$
\left\|\mathcal{M}^{\varepsilon} u\right\|_{L^{\infty}(\partial \Omega)} \leq C\|f\|_{L^{r}(M)} .
$$

Interpolation between (2.75) and (2.76) yields that for any $r>n / 2$ and any $r^{\prime}(n-1) / n<q<\infty$ there exist $\varepsilon=\varepsilon(p, q)>0$ and $\delta=\delta(p, q)>0$ such that

$$
\left\|\mathcal{M}^{\varepsilon} u\right\|_{L^{q}(\partial \Omega)} \leq C(p, q)\|f\|_{L^{r}(M)} \sigma(A)^{1 / q+\delta} .
$$

From this part (b) follows. Part (c) if $1 \leq q<(n-1) /(n-2)$ is based on modification of the estimate (2.75). We get

$$
\left\|\mathcal{M}^{\varepsilon} u\right\|_{L^{q}(\partial \Omega)} \leq\left\|v^{1}\right\|_{L^{q}(\partial \Omega)}+\left\|v^{2}\right\|_{L^{q}(\partial \Omega)} \leq C\left(\|f\|_{L^{r}} \sigma(A)^{1 / q+\delta}+\|f g\|_{L^{1}(\Omega)}\right),
$$

for some $\varepsilon=\varepsilon(r, q)>0$. Here $\delta=\delta(r, q)>0$ if $q>1, \delta=0$ if $q=1$. Once again (2.78) is crucial. The rest goes as in Theorem 3.5 of [3].

Proof of Theorem 2.3. We have almost all the required ingredients in place. Notice that if $r$ is close to $n$ ( $n / 2$, respectively), Proposition 2.6 gives us results that are almost optimal, in the sense that the improvement in Theorem 2.3 is minimal. On the other hand, if $r=\infty$, Theorem 2.3 is much stronger than Proposition 2.6. Once again, it is enough to prove (a) and (b) on the level of estimates of a type (2.77) or (2.78) for the function $h=f g$, where $f \in L^{r}(\Omega)$ and $g \in L^{\infty}(\Omega),|g(x)| \leq 1$, and $\operatorname{supp} g \subset \operatorname{Pim}(A)$ for some $A \subset \partial \Omega$. We start with (a).

Consider the map

$$
T_{g}: f \mapsto \mathcal{M}^{1+\delta} u,
$$

defined by $u=L^{-1}(\widetilde{f g}) . T_{g}$ is continuous and sublinear, with estimates

$$
\begin{aligned}
T_{g}: L^{n+\varepsilon}(\Omega) \rightarrow L^{p}(\partial \Omega), & \left\|\mathcal{M}^{1+\delta} u\right\|_{L^{p}(\partial \Omega)} \leq C\|g\|_{\mathcal{D}^{0, p}}\|f\|_{L^{n+\varepsilon}(\Omega)}, \\
T_{g}: L^{\infty}(\Omega) \rightarrow L^{s}(\partial \Omega), & \left\|\mathcal{M}^{1+\delta} u\right\|_{L^{s}(\partial \Omega)} \leq C\|g\|_{\mathcal{D}^{0, p}}\|f\|_{L^{\infty}(\Omega)},
\end{aligned}
$$

for any $s<(n-1) /((n-1) / p-1)$ and $p \leq n-1$. Here $\delta=\delta(\varepsilon)>0$, and $\varepsilon>0$ is arbitrarily small.

Marcinkiewicz's interpolation theorem gives that $T_{g}$ is bounded as a map from $L^{r}(\Omega)$ to $L^{\widetilde{q}-\delta^{\prime}}(\partial \Omega)$, where the number $1 / \widetilde{q}$ is given by the right-hand side of (2.11) and $\delta^{\prime}=\delta^{\prime}\left(s_{0}\right)>0$ is some number going to zero as $s \rightarrow(n-1) /((n-1) / p-1)$. From this (2.12) follows. 
If $p>n-1$, the situation is somewhat more complicated. Here, if we interpolate between (2.58) and (2.60) and then use (3.84) of [3] and Marcinkiewicz's interpolation theorem, we eventually get that for any $q \geq(n-1) /(n-2), r>n$, and any $\varepsilon^{\prime}>0$ there exist $\delta=\delta\left(q, r, \varepsilon^{\prime}\right)>0$ and a constant $C=C\left(q, r, \varepsilon^{\prime}\right)$ such that

$$
\left\|\mathcal{M}^{1+\delta} u\right\|_{L^{q}(\partial \Omega)} \leq C\|f\|_{L^{r}(M)} \sigma(A)^{1 / q-(n / r-1) /(n-1)-\varepsilon^{\prime}} .
$$

From this (2.12) follows.

The proof of (b) goes similarly. For $(n-1) /(n-2) \leq q<(n-1) /(n-3)$ we use Marcinkiewicz's interpolation as in $(2.80)$. For $q \geq(n-1) /(n-3)$ we interpolate between

$$
\left\|\mathcal{M}^{\delta} u\right\|_{L^{q}(\partial \Omega)} \leq C\|f\|_{L^{\infty}} \sigma(A)^{1 / q+2 /(n-1)-\varepsilon}
$$

and

$$
\left\|\mathcal{M}^{\delta} u\right\|_{L^{q}(\partial \Omega)} \leq C\|f\|_{L^{n / 2+\varepsilon}} \sigma(A)^{1 / q},
$$

for any $\varepsilon>0$ and some $\delta=\delta(q, \varepsilon)>0$. This gives that for any $q \geq(n-1) /(n-3)$, $r>n / 2$, and any $\varepsilon^{\prime}>0$ there exist $\delta=\delta\left(q, r, \varepsilon^{\prime}\right)>0$ and a constant $C=C\left(q, r, \varepsilon^{\prime}\right)$ such that

$$
\left\|\mathcal{M}^{\delta} u\right\|_{L^{q}(\partial \Omega)} \leq C\|f\|_{L^{r}} \sigma(A)^{1 / q-(n / r-2) /(n-1)-\varepsilon^{\prime}} .
$$

The other case to consider is $r(n-1) /(r-1) n \leq q<(n-1) /(n-2)$, which has to be considered only for $n / 2<r \leq n$, since for $r>n$ the result follows from part (a) by the embedding theorem. Fix $\varepsilon>0$. What we have established so far gives

$$
\begin{aligned}
\left\|\mathcal{M}^{\delta} u\right\|_{L^{(n-1) /(n-3)-\varepsilon}(\partial \Omega)} & \leq C\|f\|_{L^{n} \sigma(A)^{(n-2) /(n-1)},} \\
\left\|\mathcal{M}^{\delta} u\right\|_{L^{(n-1) /(n-2)}(\partial \Omega)} & \leq C\|f\|_{L^{n / 2+\varepsilon}} \sigma(A)^{(n-2) /(n-1)} .
\end{aligned}
$$

Hence interpolation gives, for $n / 2<r \leq n$ and any $\varepsilon^{\prime}>0$,

$$
\left\|\mathcal{M}^{\delta} u\right\|_{L^{(n-1) /(n-4+n / r)-\varepsilon^{\prime}(\partial \Omega)}} \leq C\|f\|_{L^{r} \sigma(A)^{(n-2) /(n-1)}} .
$$

for some $\delta=\delta\left(\varepsilon^{\prime}\right)>0$.

On the other hand, from (2.12) for $p=1$ we have

$$
\left\|\mathcal{M}^{\delta} u\right\|_{L^{(n-1) /(n-2)-\varepsilon^{\prime}(\partial \Omega)}} \leq C\|f\|_{L^{n+\varepsilon} \sigma(A)},
$$

where $\varepsilon, \varepsilon^{\prime}>0$ are arbitrarily small. Marcinkiewicz's interpolation between the second line of (2.85) and (2.87) consequently gives

$$
\left\|\mathcal{M}^{\delta} u\right\|_{L^{(n-1) /(n-2)-\varepsilon^{\prime}(\partial \Omega)}} \leq C\|f\|_{L^{r} \sigma(A)^{1-(n / r-1) /(n-1)},}
$$

where $\varepsilon^{\prime}>0$ is arbitrarily small.

Finally, for $f$ fixed we can interpolate between (2.86) and (2.88). This gives that for any $(n-1) /(n-2) \leq q<(n-1) /(n-4+n / r)$ we have

$$
\left\|\mathcal{M}^{\delta} u\right\|_{L^{q}(\partial \Omega)} \leq C\|f\|_{L^{r}} \sigma(A)^{1 / q-(n / r-2) /(n-1)-\varepsilon^{\prime}}
$$

for any $\varepsilon^{\prime}>0$ and some $\delta=\delta\left(\varepsilon^{\prime}\right)>0$. This is the same statement as (2.84).

The last case we have to deal with is $q<(n-1) /(n-2)$. Recall $(2.43)$. It follows that $\mathcal{M}^{\delta} u$ can be bounded from above by $v^{1}+v^{2}$, where $v^{1}, v^{2}$ have the same meaning as in (2.66) and (2.69). Crucially, it follows from (2.69) that

$$
\left\|v^{2}\right\|_{L^{q}(\partial \Omega)} \leq C\|f g\|_{L^{1}},
$$

for any $q<(n-1) /(n-2)$. 
It remains to bound $v^{1}$. Consider instead of $T_{g}$ the mapping

$$
\widetilde{T_{g}}: f \mapsto v^{1} .
$$

Mappings $T_{g}$ and $\widetilde{T_{g}}$ have similar properties, i.e., both are sublinear, and we can establish estimates similar to $(2.89)$ for $\widetilde{T_{g}}$. We leave the details to the reader, and just state the result. We get that

$$
\left\|\widetilde{T_{g}} f\right\|_{L^{q}(\partial \Omega)}=\left\|v^{1}\right\|_{L^{q}(\partial \Omega)} \leq C\|f\|_{L^{r}} \sigma(A)^{1 / q-(n / r-2) /(n-1)-\varepsilon^{\prime}},
$$

for $q \geq(n-1) /(n-3-n / r)$ and $\varepsilon^{\prime}>0$ arbitrarily small. From this Theorem 2.3 follows.

At this stage it would be possible to state results similar to Theorems 3.6, 3.7 and 3.8 in 3 . We instead choose to develop more on the Dirichlet problem for the equation $L u=f$, and only after that state the analogues of the theorems mentioned above.

\section{Dirichlet PROBlem FOR The SCAlar EQUATION}

In this section we want to combine results on general second-order scalar strongly elliptic linear equations contained in section 3 of [19] with results from 21] that allow us to consider less smooth coefficients and with the new development contained in the previous section of this paper. In addition, we prove a uniform estimate for the solutions of such equations. This estimate (3.7) will be crucial in the subsequent sections. The main result is contained in Theorem 3.1.

As in the previous section, let $L$ be a general second-order, strongly elliptic, formally selfadjoint scalar operator acting on real functions on a compact Riemannian manifold $M$ of dimension $n \geq 3$. That is, in local smooth coordinates $U \subset M$, (2.17) simplifies to

$$
\left.L\right|_{U}=\sum_{j, k} \frac{\partial}{\partial x_{j}} a_{j k} \frac{\partial}{\partial x_{k}}+\sum_{j} b_{j} \frac{\partial}{\partial x_{j}}+c .
$$

In [19], section 3 , it is assumed that

$$
a_{j k} \in C^{1+\gamma}, b_{j} \in H^{1, r}, c \in L^{r},
$$

for some $\gamma>0$ and $r>n=\operatorname{dim} M$. It was also assumed that the metric tensor on $M$ has regularity $H^{2, r}$ for some $r>n$.

As has been shown in 21], the assumption on the coefficients $a_{j k}$ and the metric tensor $g$ is unnecessarily strict in the scalar case for the Laplace-Beltrami operator. The theory there extends in a straightforward way for the operator (3.1). Hence we are going to assume that

$$
a_{j k} \in \operatorname{Lip}, b_{j} \in H^{1, r}, c \in L^{r / 2},
$$

for some $r>n=\operatorname{dim} M$. We will also assume that the metric tensor $g$ on $M$ is Lipschitz.

Theorem 3.1. Let $M$ be a smooth $n$-dimensional Riemannian manifold $(n \geq 3)$ whose metric tensor $g$ is Lipschitz. Let $L$ be a strongly elliptic, (formally) selfadjoint, negative definite second-order differential operator acting on functions on $M$ that can be locally written as (3.1) with coefficients satisfying

$$
a_{j k} \in \operatorname{Lip}, b_{j} \in H^{1, s}, c \in L^{s / 2},
$$


for some $s>n$. Let $\Omega \subset M$ be a connected Lipschitz domain on $M$.

There exists $\varepsilon=\varepsilon(\Omega, L, s)>0$ such that the following is true: If $2-\varepsilon<p \leq \infty$ and $1 \leq q \leq \infty$ satisfy

$$
\frac{1}{p}>\frac{1}{q}+\frac{2}{n-1}\left(\frac{n}{2 r}-1\right),
$$

for some $r>n / 2$, then, given any $g \in L^{p}(\partial \Omega), f \in \mathcal{L}_{r}^{q}(\Omega)$, and $V \geq 0, V \in$ $L^{s / 2}(\Omega)$, the Dirichlet boundary problem

$$
(L-V) u=f, \text { in } \Omega,\left.\quad u\right|_{\partial \Omega}=g, \quad u \in \mathcal{D}^{0, p},
$$

has a unique solution. The trace of $u$ on $\partial \Omega$ is taken in the nontangential a.e. sense. Moreover, there is a constant $C=C(L, p, \Omega)>0$, independent of $V$, such that

$$
\|u\|_{\mathcal{D}^{0, p}} \leq C\left(\|f\|_{\mathcal{L}_{r}^{q}(\Omega)}+\|g\|_{L^{p}(\partial \Omega)}\right) .
$$

If $\Omega$ is $C^{1}$, the claim is true for any $1<p \leq \infty$ as long as $1 / s+(n-1) / n p \leq 1$.

Proof. We first deal with the issue of the existence of a solution. Assume for a moment that $f=0$. If we also assume that $c$ in (3.4) and $V$ in (3.6) are more regular and both belong to $L^{s}(\Omega)$ for some $s>n$, then for $2-\varepsilon<p<2+\varepsilon$ the existence and uniqueness of a solution to (3.6) follows from Theorem 3.1 of [19], including the estimate (3.7), except the fact that the constant $C>0$ in this estimate might depend on $V$.

Indeed, the fact that $L$ is strongly elliptic and negative definite implies that $L-V$ satisfies a nonsingularity hypothesis relative to any domain $D \subset M$ as long as $V \geq 0$. Recall that an operator $L$ satisfies the nonsingularity hypothesis relative to a Lipschitz domain $D$ (possibly all of $M$ ), provided

$$
u \in H_{0}^{1,2}(D), L u=0 \text { in } D \Longrightarrow u=0 \text { in } D .
$$

This means that all assumptions of Theorem 3.1 of [19] are satisfied. It turns out that we can lower the assumptions on $c$ and $V$, and the argument in 19 still holds. As long as $s>n / 2$ the operator $(V+c): H^{1+\alpha, p}(M) \rightarrow H^{-1+\alpha, p}(M)$ is well defined and compact; hence $L-V$ is a compact perturbation of the operator $L-c$. From this our claim follows. There is also an alternative argument based on the estimate (3.7). We present it later in this proof.

On the other hand, the existence for $p=\infty$ follows from the maximum principle. One can actually deal with a more general class of domains than just Lipschitz. Now we can interpolate. The map

$$
T: g \mapsto \mathcal{M}^{0} u,
$$

where $u$ is determined by the equation (3.6), is well defined, continuous and sublinear on $L^{2-\varepsilon}(\partial \Omega)$ (by Theorem 3.1 of [19]) and also on $L^{\infty}(\partial \Omega)$. Hence Marcinkiewicz's interpolation theorem gives us that $T$ is well defined and bounded on $L^{p}(\partial \Omega)$ for any $2-\varepsilon<p \leq \infty$. In the case the domain is $C^{1}$ the existence and uniqueness can be established using layer potentials for any $1<p<\infty$. See e.g. 4 for details.

Now we deal with the case when $f \neq 0$. By Theorem 2.3 we see that $h=$ $(L-V)^{-1} \tilde{f} \in \mathcal{D}^{\delta, p}$ for some $\delta>0$, and also $\|h\|_{\mathcal{D}^{\delta, p}} \leq C\|f\|_{\mathcal{L}_{r}^{p}(\Omega)}$. Now, if $w$ solves $(L-V) w=0$ in $\Omega$ and $\left.w\right|_{\partial \Omega}=g-\left.h\right|_{\partial \Omega}$, then clearly $u=w+h$ solves (3.6). The fact that $\left.h\right|_{\partial \Omega} \in L^{p}(\partial \Omega)$ follows from Theorem 2.5 of [3]. 
Next, we want to establish the uniform estimate (3.7). Let us introduce the following notation. We denote by $L_{V}$ the operator

$$
L_{V}=L-V,
$$

and by $u_{V}$ the solution to the corresponding Dirichlet problem

$$
L_{V} u_{V}=(L-V) u_{V}=f, \text { in } \Omega,\left.\quad u_{V}\right|_{\partial \Omega}=g, \quad u_{V} \in \mathcal{D}^{0, p} .
$$

If $V=0$ is the function identically zero on $\Omega$, the solution to the problem (3.11) is now denoted by $u_{0}$. The claim is that for $f, g$ as in Theorem 3.1 and $V \geq 0$, $V \in L^{s}(\Omega)$, for some $s>n$, there is a constant $C>0$, independent of $V$, such that

$$
\left\|u_{V}\right\|_{\mathcal{D}^{0, p}} \leq C\left(\|f\|_{\mathcal{L}_{r}^{q}(\Omega)}+\|g\|_{L^{p}(\partial \Omega)}\right) .
$$

The proof requires several steps. First, for any function $h: \Omega \rightarrow \mathbb{R}$ let us introduce

$$
h^{+}=\max \{h, 0\} \quad \text { and } \quad h^{-}=\max \{-h, 0\} .
$$

Clearly $h^{+}, h^{-} \geq 0, h=h^{+}-h^{-}$and $\operatorname{supp} h^{+} \cap \operatorname{supp} h^{-}=\emptyset$. Following (3.11), let us denote by $u_{V}^{(+)}$the solution to

$$
L_{V} u_{V}^{(+)}=(L-V) u_{V}^{(+)}=-f^{-}, \text {in } \Omega,\left.\quad u_{V}^{(+)}\right|_{\partial \Omega}=g^{+}, \quad u_{V}^{(+)} \in \mathcal{D}^{0, p},
$$

and by $u_{V}^{(-)}$the solution to

$$
L_{V} u_{V}^{(-)}=(L-V) u_{V}^{(-)}=-f^{+}, \text {in } \Omega,\left.\quad u_{V}^{(-)}\right|_{\partial \Omega}=g^{-}, \quad u_{V}^{(-)} \in \mathcal{D}^{0, p} .
$$

Clearly $u_{V}=u_{V}^{(+)}-u_{V}^{(-)}$. Also, since

$$
\|f\|_{\mathcal{L}_{r}^{p}(\Omega)}=\left\|f^{+}\right\|_{\mathcal{L}_{r}^{p}(\Omega)}+\left\|f^{-}\right\|_{\mathcal{L}_{r}^{p}(\Omega)}, \quad\|g\|_{L^{p}(\partial \Omega)}=\left\|g^{+}\right\|_{L^{p}(\partial \Omega)}+\left\|g^{-}\right\|_{L^{p}(\partial \Omega)},
$$

it follows that the existence of a uniform constant $C>0$ for which (3.12) holds would follow, provided we show that

$$
0 \leq u_{V}^{(+)} \leq u_{0}^{(+)} \text {and } 0 \leq u_{V}^{(-)} \leq u_{0}^{(-)} .
$$

(3.17) is essentially a consequence of the maximum principle. It (3.17) suffices to show only the first part of (3.17), since the other one is very similar.

First, we want to argue that $u_{V}^{(+)} \geq 0$. Assume the contrary, i.e., for some $x \in \Omega, u_{V}^{(+)}(x)<0$. We approximate the function $g^{+}$by a sequence $\left(g_{i}\right)_{i \in \mathbb{N}}$ of $C(\partial \Omega)$ functions and $f^{-}$by a sequence $\left(f_{i}\right)_{i \in \mathbb{N}}$ of $L^{\infty}(\Omega)$ functions such that

$$
\begin{aligned}
g_{i} & \rightarrow g^{+} \text {in } L^{p}(\partial \Omega), & 0<g_{i}, \\
f_{i} & \rightarrow f^{-} \text {in } \mathcal{L}_{r}^{q}(\Omega), & 0 \leq f_{i} .
\end{aligned}
$$

(If $r=\infty$ we instead find $\left(f_{i}\right)_{i \in \mathbb{N}}$ such that $f_{i} \rightarrow f$ in $\mathcal{L}_{s}^{q}(\Omega)$ for any $s<\infty$.) Continuity in the variables $f$ and $g$ implies that there is an index $i \in \mathbb{N}$ such that $u(x)=u_{V}^{i,(+)}(x)<0$, where $u=u_{V}^{i,(+)}$ solves

$$
L_{V} u=(L-V) u=-f_{i}^{-}, \text {in } \Omega,\left.\quad u\right|_{\partial \Omega}=g_{i}, \quad u \in \mathcal{D}^{0, p} .
$$

The important point is that the solution $u$ to (3.19) belongs to $C(\bar{\Omega}) \cap C_{\text {loc }}^{1}(\Omega)$, since the boundary data are continuous and $f_{i} \in L^{\infty}(\Omega)$. Let $\mathcal{O}$ be the open connected component of the set $\{y \in \Omega ; u(y)<0\}$ that contains the point $x$. 
Clearly $\mathcal{O} \subset \subset \Omega$, since on the boundary $u>0$. It follows that $u \in C^{1}(\mathcal{O})$. Define the function $v: M \rightarrow \mathbb{R}$ by

$$
v(y)= \begin{cases}u(y), & \text { for } y \in \mathcal{O}, \\ 0, & \text { otherwise }\end{cases}
$$

It follows that $v \in \operatorname{Lip}(M) \subset H^{1,2}(M)$. Now $L_{V} v=-f_{i}^{-} \leq 0$ on $\mathcal{O}$, and $L_{V} v=0$ otherwise. Hence

$$
\left(L_{V} v, v\right)=-\int_{\mathcal{O}} f_{i}^{-}(y) v(y) d \operatorname{Vol}(y) \geq 0 .
$$

This is a contradiction, since $L_{V}$ is negative definite and $v \neq 0$.

Similarly, $u_{V}^{(+)} \leq u_{0}^{(+)}$holds for the same reasons. The difference $w=u_{0}^{(+)}-u_{V}^{(+)}$ solves the PDE

$$
L w=-V u_{V}^{(+)} \leq 0, \text { in } \Omega,\left.\quad w\right|_{\partial \Omega}=0, \quad w \in \mathcal{D}^{0, p} .
$$

So, the same argument as above implies that $w \geq 0$ in $\Omega$. This proves (3.17).

As we indicated above, the estimate (3.7) can be used to prove the existence of a solution for $V \in L^{s}(\Omega), s>n / 2$, knowing only that the solution exists and is unique for $V \in L^{\infty}(\Omega)$. We present this simple argument here.

Assume therefore that $V \in L^{s}(\Omega)$ for some $s>n / 2$. Then we can find a sequence of $L^{\infty}$ functions $\left(V_{i}\right)_{i \in \mathbb{N}}$ such that

$$
V_{i} \rightarrow V \quad \text { in } L^{s}(\Omega) \text { as } i \rightarrow \infty, \quad 0 \leq V_{i}, \quad i=1,2, \ldots
$$

With $f, g$ as in Theorem 3.1, for each $i \in \mathbb{N}$ there is a unique function $u_{i}$ solving

$$
\left(L-V_{i}\right) u_{i}=f, \text { in } \Omega,\left.\quad u_{i}\right|_{\partial \Omega}=g, \quad u_{i} \in \mathcal{D}^{0, p} .
$$

We claim that the sequence $\left(u_{i}\right)_{i \in \mathbb{N}}$ is Cauchy in $\mathcal{D}^{0, p}$ and denote its limit by $u$. To see this, consider the PDE satisfied by the difference $w_{i j}=u_{i}-u_{j}$. We get

(3.25) $\left(L-V_{i}\right) w_{i j}=K_{i j}, \quad$ where $K_{i j}=\left(V_{j}-V_{i}\right) u_{j},\left.\quad w_{i j}\right|_{\partial \Omega}=0, \quad w_{i j} \in \mathcal{D}^{0, p}$.

Clearly, (3.12) and (3.24) give us that all $u_{i}$ are uniformly bounded in the $\mathcal{D}^{0, p}$ norm. This together with (3.23) implies that

$$
\left\|K_{i j}\right\|_{\mathcal{L}_{s}^{p}(\Omega)} \rightarrow 0, \quad \text { as } i, j \rightarrow \infty,
$$

provided $p>s(n-1) /(s-1) n$. This can always be guaranteed by modifying $\varepsilon$ in the statement of Theorem 3.1, for $s$ close to $n / 2$. Now (3.12) used on equation (3.25) gives

$$
\left\|w_{i j}\right\|_{\mathcal{D}^{0, p}} \leq C\left\|K_{i j}\right\|_{\mathcal{L}_{s}^{p}(\Omega)} \rightarrow 0, \quad \text { as } i, j \rightarrow \infty,
$$

where the constant $C$ does not depend on $i, j$. This establishes our claim. It also follows that the limit $u \in \mathcal{D}^{0, p}$ satisfies the boundary condition $\left.u\right|_{\partial \Omega}=g$. Now, taking the limit in (3.24), we get that, in the sense of distribution,

$$
L_{V} u=(L-V) u=f .
$$

Finally, Proposition 2.1 of [19] gives that actually $u \in C_{\text {loc }}^{\delta}(\Omega)$ for some small $\delta>0$. Notice also that (3.12) remains valid even for this more general $V$, with the same constant $C>0$ (independent of $V$ ).

It remains to establish uniqueness of the constructed solution $u$ to (3.28) based on uniqueness for $V \in L^{\infty}(\Omega)$. 
Assume that the equation (3.6) has two solutions, $u_{1}$ and $u_{2}$. Using the sequence $\left(V_{i}\right)_{i \in \mathbb{N}}$ from (3.23), it follows that for each $i \in \mathbb{N}$, the difference $w=u_{1}-u_{2}$ solves the PDE

$$
\left(L-V_{i}\right) w=h_{i}, \text { in } \Omega,\left.\quad w\right|_{\partial \Omega}=0, \quad w \in \mathcal{D}^{0, p},
$$

where $h_{i}=\left(V-V_{i}\right) w \in \mathcal{L}_{s}^{p}(\Omega)$. For given $h_{i}$ the solution to the equation (3.29) is unique, and moreover (3.7) gives us that

$$
\|w\|_{\mathcal{D}^{0, p}} \leq C\left\|h_{i}\right\|_{\mathcal{L}_{s}^{p}(\Omega)} .
$$

The final point is that the norm of $h_{i}$ goes to 0 as $i \rightarrow \infty$, since $V-V_{i} \rightarrow 0$ in the $L^{s}(\Omega)$ norm. So (3.30) implies that $w=0$, i.e., $u_{1}=u_{2}$.

Corollary 3.2. Fix $f$ and $g$ as in Theorem 3.1. The map

$$
V \mapsto u_{V}
$$

where $u=u_{V}$ is the solution to (3.6), is a continuous map from $L^{s / 2}(\Omega) \cap\{V \geq 0\}$ to $\mathcal{D}^{0, p}$. Here $s$ and $p$ are as in Theorem 3.1.

Proof. Consider $V_{1}, V_{2} \in L^{s / 2}(\Omega)$, both $\geq 0$. Denote by $u_{1}, u_{2}$ the corresponding solutions to (3.6). Then the difference $w=u_{1}-u_{2}$ solves

$$
\left(L-V_{1}\right) w=\left(V_{1}-V_{2}\right) u_{2},\left.\quad w\right|_{\partial \Omega}=0, \quad w \in \mathcal{D}^{0, p} .
$$

It follows from (3.7) that

$$
\left\|u_{2}\right\|_{\mathcal{D}^{0, p}} \leq C\left(\|f\|_{\mathcal{L}_{r}^{q}(\Omega)}+\|g\|_{L^{p}(\partial \Omega)}\right)
$$

and hence

$$
\left\|\left(V_{1}-V_{2}\right) u_{2}\right\|_{\mathcal{L}_{s / 2}^{p}(\Omega)} \leq C\left(\|f\|_{\mathcal{L}_{r}^{q}(\Omega)}+\|g\|_{L^{p}(\partial \Omega)}\right)\left\|V_{1}-V_{2}\right\|_{L^{s / 2}(\Omega)} .
$$

Again by (3.7) (applied to (3.32)) we now get

$$
\left\|u_{1}-u_{2}\right\|_{\mathcal{D}^{0, p}}=\|w\|_{\mathcal{D}^{0, p}} \leq C\left(\|f\|_{\mathcal{L}_{r}^{q}(\Omega)}+\|g\|_{L^{p}(\partial \Omega)}\right)\left\|V_{1}-V_{2}\right\|_{L^{s / 2}(\Omega)} .
$$

Hence, if $\left\|V_{1}-V_{2}\right\|_{L^{s / 2}(\Omega)}$ is small, then also $\left\|u_{1}-u_{2}\right\|_{\mathcal{D}^{0, p}}$ is small.

\section{Dirichlet Semilinear elliptic Boundary problems, the $L^{p}$ Results}

In the previous two sections we have developed enough tools to take on the semilinear problem outlined in the introduction. We keep the same assumptions as in the previous sections. Namely, $M$ is a smooth, compact Riemannian manifold, $\operatorname{dim} M=n \geq 3$, with Lipschitz metric tensor. The set $\Omega \subset M$ is open and connected, with Lipschitz boundary. Let $L$ be as before, a strongly elliptic, (formally) selfadjoint, negative definite second-order differential operator acting on functions on $M$ that can be locally written as (3.1) with coefficients satisfying (3.4), for some $s>n$.

As in [3], we are interested in finding a solution $u$ to the equation

$$
L u-a(x, u) u=f, \quad \text { in } \Omega,\left.\quad u\right|_{\partial \Omega}=g, \quad u \in \mathcal{D}^{0, p},
$$

where $g \in L^{p}(\partial \Omega), f$ and $a$ are given. We assume that the function $a(x, u) \geq 0$ is Carathéodory, i.e., measurable in $x$ and continuous in $u$.

Clearly, there has to be a certain growth condition placed on the function $a$ in the variable $u$. This is true at least in the case when we consider a general boundary problem. As we will see in the next section, in certain special cases such as positive solutions, etc., the growth in the variable $u$ does not have to be restricted. 
In the paper [3] we imposed the following conditions on $a$ :

$$
\begin{aligned}
& a(x, u) \in L^{\infty}(\Omega \times \mathbb{R}), \quad \text { provided } p<\infty, \\
& \sup _{\substack{x \in \Omega \\
u \in[-M, M]}} a(x, u)<\infty, \quad \text { for all } M>0, \quad \text { provided } p=\infty \text {. }
\end{aligned}
$$

We are not going to improve the condition much for $p=\infty$, but we will allow polynomial growth in $u$ for $p<\infty$, which is a major improvement over (4.2).

Let us therefore assume that $a(x, u)$ is a function such that

$$
0 \leq a(x, u) \leq k_{1}(x)+k_{2}(x)|u|^{m},
$$

where the functions $k_{1}, k_{2} \geq 0$ and the number $m$ will be specified later. The number $m$ should depend on $p$; we will see that $m \rightarrow \infty$ as $p \rightarrow \infty$. We begin with the following auxiliary lemma.

Lemma 4.1. For any $1 \leq p<\infty$, if $v: \Omega \rightarrow \mathbb{R}$ belongs to $v \in \mathcal{D}^{0, p} \cap C_{\mathrm{loc}}(\Omega)$, then $v \in L^{p n /(n-1)}(\Omega)$, and, for some $C=C(p)>0$,

$$
\|v\|_{L^{p n /(n-1)}(\Omega)} \leq C\|v\|_{\mathcal{D}^{0, p}} .
$$

Corollary 4.2. Let $1 \leq p \leq \infty$, and assume a(x,u) satisfies (4.3) for $k_{1} \in L^{s}(\Omega)$ and $k_{2} \in L^{q}(\Omega)$, where

$$
0 \leq \frac{1}{q}<1-\frac{m}{p}\left(1-\frac{1}{n}\right) \quad \text { and } \quad \frac{m}{p}\left(1-\frac{1}{n}\right)+\frac{1}{q} \leq \frac{1}{s} \leq 1 .
$$

Then the map $\mathcal{T}: \mathcal{D}^{0, p} \cap C_{\text {loc }}(\Omega) \rightarrow L^{s}(\Omega)$ defined by

$$
v \mapsto a(., v(.))
$$

is well defined and continuous.

Proof of Lemma 4.1. Let us recall the decomposition (3.63)-(3.68) of [3]. It follows that the function $v$ can be (3.63)-(3.68) written as $v=\widetilde{v}+\sum_{i=0}^{\infty} v_{i}$, where

$$
\begin{aligned}
& \operatorname{supp} \widetilde{v} \subset \subset \Omega, \quad\|\widetilde{v}\|_{L^{\infty}(\Omega)} \leq\|v\|_{\mathcal{D}^{0, p}}, \\
& \text { supp } v_{i} \subset \operatorname{Pim}\left(A_{i}\right), \quad A_{i}=\left\{x \in \partial \Omega: \mathcal{M}^{0} v>i^{1 / p}\right\}, \quad i=0,1,2, \ldots, \\
& \text { and }\left|v_{i}\right| \leq(i+1)^{1 / p}-i^{1 / p}, \quad i=0,1,2, \ldots
\end{aligned}
$$

Also,

$$
\sum_{i=0}^{\infty} \sigma\left(A_{i}\right) \approx\left\|\mathcal{M}^{0} v\right\|_{L^{p}(\partial \Omega)}^{p} \leq\|v\|_{\mathcal{D}^{0, p}}^{p} .
$$

It follows that we can ignore the piece $\widetilde{v}$, since it belongs to $L^{\infty}$. We claim that for any $x \in \Omega$ and $s \geq 1$,

$$
\left(\sum_{i=0}^{\infty} v_{i}(x)\right)^{s} \leq \sum_{i=0}^{\infty}\left[(i+1)^{s / p}-i^{s / p}\right] \mathcal{X}_{\operatorname{Pim}\left(A_{i}\right)},
$$


where $\mathcal{X}_{\operatorname{Pim}\left(A_{i}\right)}$ is the characteristic function of the set $\operatorname{Pim}\left(A_{i}\right)$. Indeed, there is an integer $j(x) \in \mathbb{N}$ such that $v_{i}(x)>0$ for $i \leq j(x)$, and $v_{i}(x)=0$ otherwise. Hence

$$
\begin{aligned}
& \left(\sum_{i=0}^{\infty} v_{i}(x)\right)^{s}=\left(\sum_{i=0}^{j(x)} v_{i}(x)\right)^{s} \leq(j(x)+1)^{s / p} \\
= & \sum_{i=0}^{j(x)}\left[(i+1)^{s / p}-i^{s / p}\right] \leq \sum_{i=0}^{\infty}\left[(i+1)^{s / p}-i^{s / p}\right] \mathcal{X}_{\operatorname{Pim}\left(A_{i}\right)},
\end{aligned}
$$

since supp $v_{i} \subset \operatorname{Pim}\left(A_{i}\right)$. It follows that

$$
\|v\|_{L^{s}(\Omega)}^{s} \leq C\left(\|v\|_{\mathcal{D}^{0, p}}^{s}+\sum_{i=0}^{\infty} i^{s / p-1} \operatorname{Vol}\left(\operatorname{Pim}\left(A_{i}\right)\right)\right) .
$$

Here we used the fact that $(i+1)^{s / p}-i^{s / p} \approx i^{s / p-1}$. Now by $(2.70)$

$$
\operatorname{Vol}\left(\operatorname{Pim}\left(A_{i}\right)\right) \leq \sigma\left(A_{i}\right)^{n /(n-1)} .
$$

Now we want to pick $s$ such that there is a constant $C$ (independent of $i$ ) for which

$$
i^{s / p-1} \sigma\left(A_{i}\right)^{1 /(n-1)} \leq C, \quad i=0,1,2, \ldots
$$

Existence of such a constant follows from the inequality

$$
i \sigma\left(A_{i}\right)=\int_{A_{i}} i d \sigma \leq \int_{\partial \Omega}\left(\mathcal{M}^{0} v\right)^{p} d \sigma \leq\|v\|_{\mathcal{D}^{0, p}}^{p} .
$$

Hence

$$
i^{1 /(n-1)} \sigma\left(A_{i}\right)^{1 /(n-1)} \leq\|v\|_{\mathcal{D}^{0, p}}^{p /(n-1)} .
$$

It follows that if we take $s=p n /(n-1)$, then (4.13) holds. This yields

$$
\begin{aligned}
\sum_{i=0}^{\infty} i^{s / p-1} \operatorname{Vol}\left(\operatorname{Pim}\left(A_{i}\right)\right) & \leq C \sum_{i=0}^{\infty} \sigma\left(A_{i}\right)\left(i^{s / p-1} \sigma\left(A_{i}\right)^{1 /(n-1)}\right) \\
& \leq C\|v\|_{\mathcal{D}^{0, p}}^{p /(n-1)} \sum_{i=0}^{\infty} \sigma\left(A_{i}\right) \leq C\|v\|_{\mathcal{D}^{0, p}}^{p n /(n-1)} .
\end{aligned}
$$

Now, we are ready for the main result.

Theorem 4.3. Let $M$ be a smooth $n$-dimensional Riemannian manifold $(n \geq 3)$ whose metric tensor $g$ is Lipschitz. Let $L$ be a strongly elliptic, (formally) selfadjoint, negative definite second-order differential operator acting on functions on $M$ that can be locally written as (3.1) with coefficients satisfying

$$
a_{j k} \in \operatorname{Lip}, b_{j} \in H^{1, s}, c \in L^{s / 2},
$$

for some $s>n$. Finally, let $\Omega \subset M$ be a connected Lipschitz domain in $M$. Then there exists $\varepsilon=\varepsilon(L, \Omega)>0$ such that the Dirichlet boundary problem

$$
L u-a(x, u) u=f \in \mathcal{L}_{r}^{q}(\Omega) \text { in } \Omega,\left.\quad u\right|_{\partial \Omega}=g \in L^{p}(\partial \Omega), \quad u \in \mathcal{D}^{0, p} .
$$

has at least one solution $u \in \mathcal{D}^{0, p} \cap C_{\text {loc }}^{\delta}(\Omega)(\delta>0)$, provided $2-\varepsilon \leq p \leq \infty$ and

(i) a is a Carathéodory function such that

$$
0 \leq a(x, u) \leq k_{1}(x)+k_{2}(x)|u|^{m},
$$


where $k_{1}, k_{2} \geq 0, k_{1} \in L^{s}(\Omega)$ for some $s>n / 2, k_{2} \in L^{t}(\Omega)$ for some $t>n / 2$, and

$$
0 \leq m<p \frac{2-n / t}{n-1} \text {. }
$$

If $p=\infty$ it suffices to assume that for any $M>0$,

$$
\sup _{u \in[-M, M]} a(x, u) \in L^{s}(\Omega) \text { for some } s=s(M)>n / 2, \quad a(x, u) \geq 0 .
$$

(ii) $g \in L^{p}(\partial \Omega)$ and $f \in \mathcal{L}_{r}^{q}(\Omega)$, where

$$
1 \leq q \leq \infty, \quad r>n / 2, \quad \frac{1}{p}>\frac{1}{q}+\frac{2}{n-1}\left(\frac{n}{2 r}-1\right) .
$$

Moreover, there is a constant $C=C(p, q, r)>0$ such that the solution $u$ satisfies the estimate

$$
\|u\|_{\mathcal{D}^{0, p}} \leq C\left(\|f\|_{\mathcal{L}_{r}^{q}(\Omega)}+\|g\|_{L^{p}(\partial \Omega)}\right)
$$

If in addition the function $b(x, u)=a(x, u) u$ satisfies

$$
0 \leq \frac{\partial}{\partial u} b(x, u) \leq k_{1}(x)+k_{2}(x)|u|^{m},
$$

with $k_{1}, k_{2}, m$ as before, then the solution $u$ to the equation (4.18) is unique. If $p=\infty$ the uniqueness follows, provided that for any $M>0$,

$$
\sup _{u \in[-M, M]} \frac{\partial}{\partial u} b(x, u) \in L^{s}(\Omega) \text { for some } s=s(M)>n / 2, \quad \frac{\partial}{\partial u} b(x, u) \geq 0 .
$$

Proof. Assume first that $p<\infty$. The idea is to consider the map

$$
T: \mathcal{D}^{0, p} \cap C_{\text {loc }}(\Omega) \rightarrow \mathcal{D}^{0, p} \cap C_{\text {loc }}(\Omega),
$$

defined as follows. For a fixed $g \in L^{p}(\partial \Omega)$ and $f \in \mathcal{L}_{r}^{q}(\Omega)(p, q, r$ are as in the statement of our theorem) we take $v=T u$ to be the solution to the linear problem

$$
L v-a(x, u) v=f \text { in } \Omega,\left.\quad v\right|_{\partial \Omega}=g, \quad v \in \mathcal{D}^{0, p} .
$$

We need to show that this map $T$ is well defined for any $u \in \mathcal{D}^{0, p} \cap C_{\text {loc }}(\Omega)$. By Theorem 3.1 it suffices to show that $V(x)=a(x, u(x)) \in L^{s}(\Omega)$ for some $s>n / 2$. This, however, follows from Corollary 4.2. By (3.7) we also have that

$$
\|v\|_{\mathcal{D}^{0, p}} \leq C\left(\|f\|_{\mathcal{L}_{r}^{q}(\Omega)}+\|g\|_{L^{p}(\partial \Omega)}\right),
$$

for any $u \in \mathcal{D}^{0, p} \cap C_{\mathrm{loc}}(\Omega)$.

Next, we need to check that the map $T$ is continuous and compact. Continuity follows directly from Corollaries 3.2 and 4.2. It remains to show compactness. Let $\left(u_{i}\right)_{i \in \mathbb{N}}$ be any bounded sequence in the norm of $\mathcal{D}^{0, p} \cap C_{\text {loc }}(\Omega)$. From (4.28) we get that $v_{i}=T u_{i}$ are also uniformly bounded in the norm. It follows that

$$
h_{i}=a\left(x, u_{i}(x)\right) v_{i}(x)
$$

belongs to $\mathcal{L}_{s}^{p}(\Omega)$, and there is a constant $C$ such that $\left\|h_{i}\right\|_{\mathcal{L}_{s}^{p}(\Omega)} \leq C$ for all $i$. Finally, notice that

$$
L v_{i}=f+h_{i} \text { in } \Omega,\left.\quad v_{i}\right|_{\partial \Omega}=g, \quad v_{i} \in \mathcal{D}^{0, p}, \quad i=1,2, \ldots
$$

Let $V_{i}=L^{-1} \widetilde{f}+L^{-1} \widetilde{h_{i}}$, where $L^{-1}$ has the same meaning as in Theorem 2.3. It follows that there are $\delta>0$ and $C>0$ such that

$$
\left\|V_{i}\right\|_{\mathcal{D}^{\delta, p}} \leq C, \quad i=1,2,3, \ldots
$$


Theorem 2.4 of $\left[3\right.$ gives that $\mathcal{D}^{\delta, p} \subset \subset \mathcal{D}^{0, p}$; hence there is a subsequence of $\left(V_{i}\right)_{i \in \mathbb{N}}$, which for simplicity we denote again by $\left(V_{i}\right)_{i \in \mathbb{N}}$, that is convergent in $\mathcal{D}^{0, p}$. Denote this limit by $V_{0}$. Also by Theorem 2.5 of [3] this subsequence can be chosen such that

$$
\left.\left.V_{i}\right|_{\partial \Omega} \rightarrow V_{0}\right|_{\partial \Omega} \text { in } L^{p}(\partial \Omega) \text { as } i \rightarrow \infty
$$

Finally, let $w_{i}, i=0,1,2, \ldots$, solve the equation

$$
L w_{i}=0 \text { in } \Omega,\left.\quad w_{i}\right|_{\partial \Omega}=g-\left.V_{i}\right|_{\partial \Omega}, \quad w_{i} \in \mathcal{D}^{0, p} .
$$

It follows from (4.32) and Theorem 3.1 that $w_{i} \rightarrow w_{0}$ in $\mathcal{D}^{0, p}$ as $i \rightarrow \infty$. Since $v_{i}=V_{i}+w_{i}$, we get that $v_{i} \rightarrow v_{0}$ in $\mathcal{D}^{0, p}$ as $i \rightarrow \infty$. Hence $T$ is compact. The Schauder fixed point theorem therefore implies that $T$ has a fixed point which is the desired solution to (4.18). The estimate (4.23) follows from (4.28).

If $p=\infty$, it suffices to assume (4.21). To see this, we modify the function $a$ exactly as in 3 . Consider a function $\psi_{M}$ defined as follows:

$$
\psi_{M}(x)= \begin{cases}x, & \text { for }|x| \leq 2 M, \\ 2 M \operatorname{sgn}(x), & \text { otherwise. }\end{cases}
$$

Here $M$ is taken to be equal to the right-hand side of the estimate (3.7). Next we solve the Dirichlet problem

$$
L u-a\left(x, \psi_{M}(u)\right) u=f \text { in } \Omega,\left.\quad u\right|_{\partial \Omega}=g, \quad u \in \mathcal{D}^{0, q} .
$$

The exact choice of $2 \leq q<\infty$ is not important. Notice that $a\left(x, \psi_{M}(u)\right) \in$ $L^{\min \{s, t\}}(\Omega)$; hence (4.19)-(4.20) hold. It follows that there is at least one solution $u$ to (4.35). We will show that this $u$ also solves the original equation.

To see this, set $V(x)=a\left(x, \psi_{M}(u)\right) \in L^{s}(\Omega)$ for some $s>n / 2$. Consider the linear problem

$$
(L-V) v=f \text { in } \Omega,\left.\quad v\right|_{\partial \Omega}=g, \quad u \in \mathcal{D}^{0, q},
$$

for any $2 \leq q \leq \infty$. Since $g \in L^{\infty}(\Omega)$, Theorem 3.1, namely the uniqueness and existence results there, gives us that there is a unique function $v \in \mathcal{D}^{0,2}$ solving (4.36), and moreover $v \in \mathcal{D}^{0, \infty}=L^{\infty}(\Omega)$. Since $u$ from (4.35) solves (4.36) and $u \in \mathcal{D}^{0,2}$, it follows that $u=v$; hence $u \in L^{\infty}(\Omega)$.

Finally, the estimate (3.7) shows that $\|u\|_{L^{\infty}(\Omega)} \leq M$. That is, $\psi_{M}(u(x))=u(x)$ for all $x \in \Omega$, and hence $u$ indeed solves (4.18).

For uniqueness, it has been shown in [3] (Example 5.3) that (4.18) might have more that one solution. Hence we need a stronger condition on the function $a$. (4.24) and (4.25) give us that. Let $u_{1}, u_{2} \in \mathcal{D}^{0, p} \cap C_{\text {loc }}(\Omega)$ be two different solutions to (4.18) with $\left.u_{1}\right|_{\partial \Omega}=\left.u_{2}\right|_{\partial \Omega}$. Then, writing

$$
a\left(x, u_{1}(x)\right) u_{1}(x)-a\left(x, u_{2}(x)\right) u_{2}(x)=V_{12}(x)\left(u_{1}(x)-u_{2}(x)\right)
$$

with

$$
V_{12}(x)=\int_{0}^{1} \frac{\partial}{\partial u} b\left(x, u_{2}(x)+t\left(u_{1}(x)-u_{2}(x)\right) d t,\right.
$$

we have $V_{12}(x) \geq 0$ and $V_{12} \in L^{s}(\Omega)$, for some $s>n / 2$. Hence, the function $w=u_{1}-u_{2}$ satisfies the PDE

$$
\left(L-V_{12}\right) w=0 \quad \text { in } \Omega,\left.\quad w\right|_{\partial \Omega}=0, \quad w \in \mathcal{D}^{0, p} .
$$


The uniqueness result from Theorem 3.1 for this linear equation guarantees that $w=0$ in $\Omega$.

Example 4.4. A good example illustrating Theorem 4.3 is the equation

$$
\Delta u-|u|^{q} u=0 \text { in } \Omega,\left.\quad u\right|_{\partial \Omega}=g \in L^{p}(\partial \Omega),
$$

for some $q \geq 0$. Theorem 4.3 gives us that the solution $u$ to the equation (4.40) exists and is unique, provided that

$$
0 \leq q<\frac{2 p}{n-1} .
$$

If $p=\infty, q$ can be taken arbitrarily large. In particular, if $\operatorname{dim} M=3$, then, given any $g \in L^{p}(\partial \Omega)(p>2-\varepsilon)$, the solution $u$ exists, provided $q<p$. As we will see later, if we consider only nonnegative solutions (i.e., $g \geq 0$ ), then the condition (4.41) is not necessary, and the solution $u \in \mathcal{D}^{0, p}$ exists for any given $q \geq 0$.

We can also extend the range of $q$ for which (4.40) is solvable to negative numbers, namely the interval $-1<q<0$. This does not follow immediately from Theorem 3.2 , and the proof requires a bit of additional work.

Denote by $\phi_{i}: \mathbb{R} \rightarrow \mathbb{R}^{+}$the function

$$
\phi_{i}(u)=\left\{\begin{array}{ll}
|u|^{q}, & \text { for }|u|>1 / i, \\
i^{-q}, & \text { otherwise },
\end{array} \quad i=1,2, \ldots .\right.
$$

Since $\phi_{i}(u) \in L^{\infty}(\mathbb{R})$, it makes sense to consider an approximate solution to the equation (4.40):

$$
\Delta u_{i}-\phi_{i}\left(u_{i}\right) u_{i}=0 \text { in } \Omega,\left.\quad u_{i}\right|_{\partial \Omega}=g \in L^{p}(\partial \Omega), \quad u_{i} \in \mathcal{D}^{0, p},
$$

for any $2-\varepsilon<p \leq \infty$. Theorem 4.3 tells us that $u_{i}$ exists and, for some $C>0$,

$$
\left\|u_{i}\right\|_{\mathcal{D}^{0, p}} \leq C\|g\|_{L^{p}(\partial \Omega)}, \quad i=1,2,3, \ldots
$$

We want to argue that $\left(u_{i}\right)_{i \in \mathbb{N}}$ has a convergent subsequence in $\mathcal{D}^{0, p}$. To see this, consider $h_{i}=\phi_{i}\left(u_{i}\right) u_{i}$. Clearly,

$$
\left|h_{i}\right|=\left|\phi_{i}\left(u_{i}\right) u_{i}\right| \leq\left|u_{i}\right|^{q+1} .
$$

Since $0<1+q<1$, it follows from (4.44) and (4.45) that for some $C>0$,

$$
\left\|h_{i}\right\|_{\mathcal{D}^{0, p}} \leq C, \quad i=1,2,3, \ldots
$$

This means $u_{i}$ solves the PDE

$$
\Delta u_{i}=h_{i} \text { in } \Omega,\left.\quad u_{i}\right|_{\partial \Omega}=g \in L^{p}(\partial \Omega), \quad u_{i} \in \mathcal{D}^{0, p} .
$$

Compare this with (4.29)-(4.30)! Hence, essentially the same argument as used in (4.29)-(4.33) gives us that a subsequence of $\left(u_{i}\right)_{i \in \mathbb{N}}$ (again denoted by $\left(u_{i}\right)_{i \in \mathbb{N}}$ ) is convergent in $\mathcal{D}^{0, p}$. Denote this limit by $u$. Clearly, $\left.u\right|_{\partial \Omega}=\left.u_{i}\right|_{\partial \Omega}=g$ almost everywhere on $\partial \Omega$. Also, for any test function $\psi \in C_{0}^{\infty}(\Omega)$,

$$
\begin{aligned}
0 & =\left(u_{i}, \Delta \psi\right)-\left(\phi_{i}\left(u_{i}\right) u_{i}, \psi\right) \\
& =\left(u_{i}, \Delta \psi\right)-\left(\left|u_{i}\right|^{q} u_{i}, \psi\right)+\left(\left|u_{i}\right|^{q} u_{i}-\phi_{i}\left(u_{i}\right) u_{i}, \psi\right), \quad i=1,2, \ldots
\end{aligned}
$$


Now, $u_{i}(x) \rightarrow u(x)$ for every $x \in \partial \Omega$, and we also have that $u_{i} \rightarrow u$ in $C_{\text {loc }}(\Omega)$. Hence clearly the first two terms on the right in (4.48) converge to $(u, \Delta \psi)-$ $\left(|u|^{q} u, \psi\right)$ as $i \rightarrow \infty$. The last term is nonzero only if $\left|u_{i}\right|<i^{-1}$. Hence we get

$$
\begin{aligned}
\lim _{i \rightarrow \infty}\left|\left(\left|u_{i}\right|^{q} u_{i}-\phi_{i}\left(u_{i}\right) u_{i}, \psi\right)\right| & \leq \lim _{i \rightarrow \infty} \int_{\left\{x \in \Omega:\left|u_{i}(x)\right|<i^{-1}\right\}} 2\left|u_{i}\right|^{q+1} \psi d \operatorname{Vol} \\
& \leq 2 \lim _{i \rightarrow \infty} i^{-(q+1)}\|\psi\|_{L^{\infty}(\Omega)} \operatorname{Vol}(\Omega)=0 .
\end{aligned}
$$

Hence:

$$
(u, \Delta \psi)-\left(|u|^{q} u, \psi\right)=0, \quad \text { for all } \psi \in C_{0}^{\infty}(\Omega),
$$

or equivalently

$$
\Delta u-|u|^{q} u=0 \quad \text { in } \Omega \text { in the sense of distributions. }
$$

This proves the existence. For uniqueness, let $u_{1}, u_{2} \in \mathcal{D}^{0, p}$ be two different solutions to the equation (4.40) for some $-1<q<0$ and $p>2-\varepsilon$. Pick any $i \in \mathbb{N}$ and consider the following two sets:

$$
\Omega_{1}=\left\{x \in \Omega:\left|u_{1}(x)\right|<i^{-1} \&\left|u_{2}(x)\right|<i^{-1}\right\}, \quad \Omega_{2}=\Omega \backslash \Omega_{1} .
$$

We claim that if $x \in \Omega_{2}$, then

$$
0 \leq \frac{\left|u_{1}(x)\right|{ }^{q} u_{1}(x)-\left|u_{2}(x)\right|^{q} u_{2}(x)}{u_{1}(x)-u_{2}(x)} \leq i^{-q} .
$$

The first inequality is clear, and the second one can be proven by considering two cases when $u_{1}(x)$ and $u_{2}(x)$ have the same signs and when the signs are different. Let

$$
\begin{aligned}
& V_{i}(x)= \begin{cases}\frac{\left|u_{1}(x)\right|^{q} u_{1}(x)-\left|u_{2}(x)\right|^{q} u_{2}(x)}{u_{1}(x)-u_{2}(x)}, & \text { for } x \in \Omega_{2}, \\
0, & \text { for } x \in \Omega_{1},\end{cases} \\
& h_{i}(x)= \begin{cases}\left.\left|u_{1}(x)\right|\right|^{q} u_{1}(x)-\left|u_{2}(x)\right|^{q} u_{2}(x), & \text { for } x \in \Omega_{1}, \\
0, & \text { for } x \in \Omega_{2} .\end{cases}
\end{aligned}
$$

It follows that $V_{i}, h_{i} \in L^{\infty}(\Omega)$ and $\left\|h_{i}\right\|_{L^{\infty}(\Omega)} \leq 2 i^{-1-q}$. If we denote by $w$ the difference $u_{1}-u_{2}$, we get that

$$
\left(\Delta-V_{i}\right) w=h_{i},\left.\quad w\right|_{\partial \Omega}=0, \quad w \in \mathcal{D}^{0, p} .
$$

This and Theorem 3.1 yield that there is $C>0$, independent of $i$, such that

$$
\|w\|_{\mathcal{D}^{0, p}} \leq C\left\|h_{i}\right\|_{L^{\infty}(\Omega)} \leq 2 C i^{-1-q} .
$$

Since $i$ can be taken arbitrarily large, it follows that $\|w\|_{\mathcal{D}^{0, p}}=0$; hence $u_{1}=u_{2}$.

\section{Boundary VALUe PROBLEMS FOR NONNEGATIVE SOLUTIONS}

Consider now a special case of the equation (4.1) when the solution $u$ does not change sign, i.e., it is either always nonnegative or nonpositive. We formulate our result only for nonnegative solutions, since the other case is analogous. The main point is that instead of the condition (4.24) we have to require (4.25) only.

Theorem 5.1. Let $M$ be a smooth $n$-dimensional Riemannian manifold $(n \geq 3)$ with Lipschitz metric tensor $g$. Let $L$ be a strongly elliptic, (formally) selfadjoint, negative definite second-order differential operator acting on functions on $M$ that can be locally written as (3.1) with coefficients satisfying (4.17) for some $s>n$. Let 
$\Omega \subset M$ be a connected Lipschitz domain in $M$. Consider the following Dirichlet boundary problem:

$$
L u-b(x, u)=f \text { in } \Omega,\left.\quad u\right|_{\partial \Omega}=g \in L^{p}(\partial \Omega), \quad u \in \mathcal{D}^{0, p},
$$

where $b(x, u)$ is a Carathéodory function, $b(x, 0)=0$ and for any $M>0$,

$$
\sup _{u \in[-M, M]} \frac{\partial}{\partial u} b(x, u) \in L^{s}(\Omega) \text { for some } s=s(M)>n / 2, \quad \frac{\partial}{\partial u} b(x, u) \geq 0 .
$$

There exists $\varepsilon=\varepsilon(L, \Omega)>0$ such that the following is true. For any $2-\varepsilon \leq$ $p \leq \infty$, if $r, q$ satisfy the conditions

$$
1 \leq q \leq \infty, \quad r>n / 2, \quad \frac{1}{p}>\frac{1}{q}+\frac{2}{n-1}\left(\frac{n}{2 r}-1\right),
$$

then, given any $g \in L^{p}(\partial \Omega), g \geq 0$, and $f \in \mathcal{L}_{r}^{q}(\Omega), f \leq 0$, there exists a unique solution $u \in \mathcal{D}^{0, p}$ to the equation (5.1). (Here the trace of $u$ on $\partial \Omega$ is taken in the nontangential a.e. sense.) Also, $u \geq 0$ and $u \in C_{\mathrm{loc}}^{\delta}(\Omega)$ for some $\delta>0$.

Moreover, there is a constant $C=C(p, q, r)>0$ such that

$$
\|u\|_{\mathcal{D}^{0, p}} \leq C\left(\|f\|_{\mathcal{L}_{r}^{q}(\Omega)}+\|g\|_{L^{p}(\partial \Omega)}\right) .
$$

Proof. Notice that the equation (5.1) does not differ from equation (4.1), since we can put

$$
a(x, u)=\int_{0}^{1} \frac{\partial}{\partial u} b(x, t u) d t .
$$

It follows that $a(x, u) u=b(x, u)$, and moreover $a(x, u)$ satisfies, for any $M>0$,

$$
\sup _{u \in[-M, M]} a(x, u) \in L^{s}(\Omega) \text { for some } s=s(M)>n / 2, \quad a(x, u) \geq 0 .
$$

Fix $f$ and $g$ as in the statement of our theorem. First we solve the following linear problem:

$$
L v=f \text { in } \Omega,\left.\quad v\right|_{\partial \Omega}=g \in L^{p}(\partial \Omega), \quad v \in \mathcal{D}^{0, p} .
$$

The solution to this problem exists by Theorem 3.1, and moreover $v \geq 0$, as follows from (3.14) and (3.17). Also,

$$
\|v\|_{\mathcal{D}^{0, p}} \leq C\left(\|f\|_{\mathcal{L}_{r}^{q}(\Omega)}+\|g\|_{L^{p}(\partial \Omega)}\right) .
$$

We solve (5.1) by constructing a sequence of approximate solutions $\left(u_{i}\right)_{i \in \mathbb{N}}$ and then letting $i \rightarrow \infty$. We take

$$
\begin{aligned}
& g_{i}(x)= \begin{cases}g(x), & \text { if } g(x) \leq i, \\
i, & \text { otherwise },\end{cases} \\
& f_{i}(x)= \begin{cases}f(x), & \text { if } \operatorname{dist}(x, \partial \Omega) \geq 1 / i \\
0, & \text { otherwise }\end{cases}
\end{aligned}
$$

Notice that $g_{i} \in L^{\infty}(\partial \Omega)$ and $f_{i} \in L^{r}(\Omega)$. Let $u_{i}$ be the solution to the nonlinear equation

$$
L u_{i}-b\left(x, u_{i}\right)=f_{i} \text { in } \Omega,\left.\quad u_{i}\right|_{\partial \Omega}=g_{i}, \quad u_{i} \in \mathcal{D}^{0, \infty} .
$$

First, we have to make sure the equation (5.10) is solvable. This is quite clear, since Theorem 4.2 applies. This theorem also gives that $u_{i} \in L^{\infty}(\Omega)$ and $u_{i}$ is uniquely 
determined since (5.2) holds. Also the same argument as used for the function $v$ shows that $u_{i} \geq 0$.

Our goal is to prove that for any $i=1,2,3, \ldots$

$$
0 \leq u_{i} \leq u_{i+1} \leq v
$$

Fix any $i \in \mathbb{N}$. The difference $w_{i}=u_{i+1}-u_{i}$ solves the PDE

$$
\left(L-V_{i}\right) w_{i}=h_{i} \text { in } \Omega,\left.\quad w_{i}\right|_{\partial \Omega}=g_{i+1}-g_{i}, \quad w_{i} \in \mathcal{D}^{0, \infty},
$$

where $h_{i}=f_{i+1}-f_{i} \in L^{r}(\Omega), h_{i} \leq 0$, and

$$
V_{i}(x)=\int_{0}^{1} \frac{\partial}{\partial u} b\left(x, u_{i}(x)+t\left(u_{i+1}(x)-u_{i}(x)\right) d t,\right.
$$

i.e., $V_{12}(x) \geq 0$ and $V_{12} \in L^{s}(\Omega)$ for some $s>n / 2$ by (5.2). Hence $w_{i} \geq 0$ by (3.14) and (3.17). Similarly, the difference $W_{i}=v-u_{i}$ solves the PDE

$$
L\left(W_{i}\right)=H_{i}=-b\left(x, u_{i}\right)+f-f_{i} \text { in } \Omega,\left.\quad W_{i}\right|_{\partial \Omega}=g-g_{i}, \quad W_{i} \in \mathcal{D}^{0, p} .
$$

Since $u_{i} \geq 0$, we have $-b\left(x, u_{i}\right) \leq 0$. Also $f-f_{i} \leq 0$, from which follows that $H_{i} \leq 0$. Moreover, $b\left(x, u_{i}\right) \in L^{\infty}(\Omega), f-f_{i} \in \mathcal{L}_{r}^{q}(\Omega)$, and therefore $H_{i} \in \mathcal{L}_{r}^{q}(\Omega)$. Finally, $\left.W_{i}\right|_{\partial \Omega} \geq 0$, which again gives that $W_{i} \geq 0$. So (5.11) holds. It follows that for any $x \in \Omega$ the number

$$
u(x)=\lim _{i \rightarrow \infty} u_{i}(x)
$$

is well defined and $0 \leq u(x) \leq v(x)$. Hence the function $u$ belongs to $\mathcal{D}^{0, p}$ and the estimate (5.4) holds. We also see that $\left.u\right|_{\partial \Omega}=g$ a.e. on $\partial \Omega$, where this trace is taken in the nontangential sense. This follows from the fact that for all $i$ we have $\left.u_{i}\right|_{\partial \Omega}=g_{i}$ a.e., $\left.v\right|_{\partial \Omega}=g$ a.e. and $g_{i} \rightarrow g$ as $i \rightarrow \infty$.

It remains to prove that

$$
L u-b(x, u)=f \text { in } \Omega
$$

in the sense of distributions. But this is easy. Take any test function $\psi \in C_{0}^{\infty}(\Omega)$. On $\mathcal{O}=\operatorname{supp} \psi \subset \subset \Omega$ we have that $\left.v\right|_{\mathcal{O}} \in L^{\infty}(\mathcal{O})$ and also $u_{i}(x) \rightarrow u(x)$ pointwise for $x \in \mathcal{O}$. From (5.10),

$$
\left(u_{i}, L^{*} \psi\right)-\left(b\left(x, u_{i}\right), \psi\right)=\left(f_{i}, \psi\right), \quad i=1,2, \ldots
$$

Hence $b\left(x, u_{i}(x)\right) \rightarrow b(x, u(x))$, and by (5.2) the sequence $\left(b\left(., u_{i}(.)\right)\right)_{i \in \mathbb{N}}$ has an $L^{s}$ $(s>n / 2)$ majorant on $\mathcal{O}$. Finally, $f_{i} \rightarrow f$ in $L^{r}(\mathcal{O})$. This means that we can pass to the limit as $i \rightarrow \infty$ in all three terms of (5.17) to get the desired result:

$$
\left(u, L^{*} \psi\right)-(b(x, u), \psi)=(f, \psi) .
$$

This proves the existence of a solution to (5.1). The proof of uniqueness goes as follows. Assume that $u_{1}$ and $u_{2}$ both solve (5.1). Consider an increasing sequence of connected Lipschitz domains $\Omega_{1} \subset \subset \Omega_{2} \subset \subset \ldots$ converging to $\Omega=\Omega_{0}$, such that the Lipschitz character of these domains is the same. That is, there is a universal constant $L>0$ such that for any $x \in \partial \Omega$ there are a neighborhood $U$ of $x$ and a smooth coordinate system on $U$ in which for each $i=0,1,2, \ldots$ we can write $U \cap \Omega_{i}$ as

$$
U \cap \Omega_{i}=\left\{x=\left(x^{\prime}, x_{n}\right) \in U: x_{n}>\varphi_{i}\left(x^{\prime}\right)\right\}
$$

(compare with (2.39)), where the functions $\varphi_{i}$ have Lipschitz constants bounded by $L$. On each domain $\Omega_{i}$ one can consider the linear Dirichlet boundary problem 
(3.6). One also gets the appropriate estimate (3.7) for $\Omega_{i}$. Crucial here is that the constant $C$ in the estimate (3.7) can be taken the same for each domain $\Omega_{i}$, i.e., independent of $i$. This follows from the fact that these domains have the same Lipschitz character. (See also [20] for more details.)

Denote $V_{i j}(x)=\left.a\left(x, u_{j}(x)\right)\right|_{\Omega_{i}}$, for $i=1,2, \ldots$ and $j=1,2$. (The function $a$ is defined by (5.5).) Since $u_{j} \in L_{\mathrm{loc}}^{\infty}(\Omega)$, clearly $V_{i j} \in L^{s}\left(\Omega_{i}\right)$, for some $s>n / 2$. It follows that $u_{j}$ restricted to $\Omega_{i}$ solves the PDE

$$
\left(L-V_{i j}\right) u_{j}=f \text { in } \Omega_{i},\left.\quad u_{j}\right|_{\partial \Omega_{i}}=g_{i j}, \quad u_{j} \in \mathcal{D}^{0, p}\left(\Omega_{i}\right),
$$

where

$$
g_{i j}=\left.u_{j}\right|_{\partial \Omega_{i}} \in L^{p}\left(\partial \Omega_{i}\right), \quad i=1,2, \ldots
$$

It is possible to identify the boundaries $\partial \Omega_{i}$ with $\partial \Omega$ via the flow of a smooth vector field $X$ on $M$, nonzero near $\partial \Omega$ and pointing inside $\Omega$ (see [4] for more details). Using this identification, it follows that

$$
g_{i j} \rightarrow g \quad \text { in } L^{p}(\partial \Omega) \text { as } i \rightarrow \infty, \quad j=1,2 .
$$

This, (5.20) and (3.7) give us, on each domain $\Omega_{i}$ for $j=1,2$,

$$
\left\|u_{j}\right\|_{\mathcal{D}^{0, p}\left(\Omega_{i}\right)} \leq C\left(\|f\|_{\mathcal{L}_{r}^{q}\left(\Omega_{i}\right)}+\left\|g_{i j}\right\|_{L^{p}\left(\partial \Omega_{i}\right)}\right) \leq C\left(\|f\|_{\mathcal{L}_{r}^{q}(\Omega)}+\|g\|_{L^{p}(\partial \Omega)}\right) .
$$

This gives that $u_{j} \in C_{\mathrm{loc}}(\Omega)$ for $j=1,2$. Interior regularity results for the equation (5.20) actually give $u_{j} \in C_{\mathrm{loc}}^{\delta}(\Omega)$, for some $\delta>0$.

The difference $w=u_{1}-u_{2}$ satisfies in $\Omega_{i}$ the PDE

$$
\left(L-\widetilde{V}_{i}\right) w=0 \text { in } \Omega_{i},\left.\quad w\right|_{\partial \Omega_{i}}=g_{i 1}-g_{i 2}, \quad w \in \mathcal{D}^{0, p}\left(\Omega_{i}\right),
$$

where

$$
\widetilde{V}_{i}(x)=\int_{0}^{1} \frac{\partial}{\partial u} b\left(x, u_{1}(x)+t\left(u_{2}(x)-u_{1}(x)\right) d t, \quad \text { for } x \in \Omega_{i} .\right.
$$

It follows that $\widetilde{V}_{i} \in L^{s}\left(\Omega_{i}\right)$ for some $s>n / 2$, and $\widetilde{V}_{i} \geq 0$. Hence, again by (3.7), we get

$$
\|w\|_{\mathcal{D}^{0, p}\left(\Omega_{i}\right)} \leq C\left\|g_{i 1}-g_{i 2}\right\|_{L^{p}\left(\partial \Omega_{i}\right)} \rightarrow 0, \quad \text { as } i \rightarrow \infty .
$$

Now, as follows from Definition 2.1, an appropriate choice of nontangetial regions $\left\{\gamma_{i}().\right\}$ for the domains $\Omega_{i}$ and $\{\gamma()$.$\} for \Omega$ implies that

$$
\|w\|_{\mathcal{D}^{0, p}(\Omega)}=\lim _{i \rightarrow \infty}\|w\|_{\mathcal{D}^{0, p}\left(\Omega_{i}\right)}=0 .
$$

Hence $u_{1}=u_{2}$, and uniqueness follows.

\section{DiRICHLET BOUNDARY PROBLEMS WITH BMO BOUNDARY DATA}

As we saw in Theorem 4.3, if $\left.u\right|_{\partial \Omega} \in L^{\infty}(\partial \Omega)$, we do not require any condition on the growth of $a(x, u)$ in the variable $u$, whereas $\left.u\right|_{\partial \Omega} \in L^{p}(\partial \Omega), p<\infty$, requires at most polynomial growth in the variable $u$.

Recall that the space BMO of bounded mean oscillations introduced by John and Nirenberg [14] is contained in all $L^{p}$ spaces for $p<\infty$ but is larger than $L^{\infty}$. Hence we can expect that the boundary condition $\left.u\right|_{\partial \Omega} \in \operatorname{BMO}(\partial \Omega)$ should allow us to solve the nonlinear problem for functions $a(x, u)$ with faster than polynomial growth in the variable $u$, but not arbitrary growth as in the $L^{\infty}$ case. This is indeed the case, as follows from Theorem 6.5. Let us start with a few definitions. 
Definition 6.1. We say that $f: \partial \Omega \rightarrow \mathbb{R}$ is a function of bounded mean oscillation, and write $f \in \mathrm{BMO}$, provided that

(a) $f$ is an integrable function on $\partial \Omega$, and

(b) there exists a constant $A$ such that, for any ball $B$ on $\partial \Omega$,

$$
\frac{1}{\sigma(B)} \int_{B}\left|f(x)-f_{B}\right| d \sigma(x) \leq A .
$$

Here $\sigma$ is the surface measure on $\partial \Omega$ and $f_{B}=\sigma(B)^{-1} \int_{B} f d \sigma(x)$.

The smallest bound $A$ for which (6.1) holds is taken to be the norm of the space and is denoted by $\|f\|_{\text {BMO }}$. Notice that the constants have zero BMO norm. To avoid this problem, we put

$$
\|f\|_{\mathrm{bmo}}=\|f\|_{L^{1}(\partial \Omega)}+\|f\|_{\mathrm{BMO}} .
$$

(6.2) satisfies all properties of a norm. The $L^{1}$ norm in (6.2) could be replaced by the $L^{q}$ norm for any $q<\infty$, giving us an equivalent norm. The space equipped with the norm (6.2) we denote by bmo or $\operatorname{bmo}(\partial \Omega)$.

Remark. The balls on the boundary $\partial \Omega$ are defined using the distance function $d(.,$.$) inherited from the manifold M$. Hence for any $x \in \partial \Omega$ and $r>0$ we can take

$$
B=B(x, r)=\{y \in \partial \Omega: d(x, y)<r\} .
$$

The paper 14] by John and Nirenberg established several important properties of bmo functions. The first one is that there is a constant $C>0$ such that for any bmo function $f$ and any $2 \leq p<\infty$

$$
\|g\|_{L^{p}(\partial \Omega)} \leq C p\|g\|_{\mathrm{bmo}},
$$

i.e., the $L^{p}$ norm of $f$ increases at most linearly as $p \rightarrow \infty$.

If a function satisfies (6.4), then the measure of the level set $\{x \in \partial \Omega ; f(x)>\alpha\}$ decays exponentially as $\alpha \rightarrow \infty$, i.e., there are positive constants $K, b$ such that

$$
\sigma(\{x \in \partial \Omega ; f(x)>\alpha\}) \leq K e^{-b \alpha} .
$$

Here $b$ on the right-hand side of (6.5) depends on the right-hand side of (6.4); namely, (6.5) works for any $b<C /\|g\|_{\text {BMO. }}$.

For reasons that will become clear later, we want to work with a space slightly larger than bmo, but satisfying (6.4). We call this space $\mathrm{LI}(\partial \Omega)$ (LI stands for linear increase). We also define the space $\mathcal{D}^{\mathrm{LI}}$ in a way that resembles Definition 2.1 .

Definition 6.2. Let $\operatorname{LI}(\partial \Omega)$ be a space of all functions $f: \partial \Omega \rightarrow \mathbb{R}$ for which

$$
\mathrm{LI}(\partial \Omega)=\left\{f: \partial \Omega \rightarrow \mathbb{R} ; \sup _{p \geq 2} p^{-1}\|f\|_{L^{p}(\partial \Omega)}<\infty\right\} .
$$

Then for any $2 \leq q<\infty$ the space $\operatorname{LI}(\partial \Omega)$ equipped with the norm

$$
\|f\|_{q}=\sup _{p \geq q} p^{-1}\|f\|_{L^{p}(\partial \Omega)}
$$

is a Banach space. Also all norms $\|\cdot\|_{q}$ are equivalent. Consider also

$$
\|f\|_{\infty}=\lim _{q \rightarrow \infty}\|f\|_{q} .
$$


Definition 6.3. Let the meaning of $\Omega \subset M,\{\gamma(x)\}_{x \in \partial \Omega}$ and $\widetilde{\Omega}$ be exactly the same as in Definition 2.1. Consider

$$
\mathcal{D}^{\mathrm{LI}}=\left\{f: \Omega \rightarrow \mathbb{R} ; \mathcal{M}^{0} f \in \operatorname{LI}(\partial \Omega)\right\} .
$$

Then

$$
\|f\|_{\mathcal{D}_{q}^{\mathrm{LI}}}=\left\|\mathcal{M}^{0} f\right\|_{q}, \quad \text { for } 2 \leq q \leq \infty,
$$

defines a norm on the space $\mathcal{D}^{\mathrm{LI}}$ for any $2 \leq q<\infty$ (a pseudonorm if $q=\infty$ ). All these norms are equivalent, and moreover the normed space $\mathcal{D}^{\mathrm{LI}}$ is complete.

Remark. The proofs of the statements in Definitions 6.2 and 6.3 are fairly straightforward. Clearly (6.4) implies that $\operatorname{bmo}(\partial \Omega) \subset \operatorname{LI}(\partial \Omega)$. We note that $\|\cdot\|_{\infty}$ and $\|\cdot\|_{\mathcal{D}_{\infty}^{\text {LI }}}$ satisfy all the properties of a norm except

$$
\|g\|_{\infty}=0 \Longrightarrow g=0 \quad \text { and } \quad\|f\|_{\mathcal{D}_{\infty}^{\mathrm{LI}}}=0 \Longrightarrow f=0 .
$$

For example, if $g \in L^{\infty}(\partial \Omega)$, then $\|g\|_{\infty}=0$. However, the number $\|g\|_{\infty}$ still carries information on $b$ in the estimate (6.5). In particular, there exists $C>0$ such that (6.5) is true for any $b$ for which

$$
b<C /\|g\|_{\infty} \text { and } g \in \operatorname{LI}(\partial \Omega) .
$$

It follows from (6.5) and (6.12) that, given any $1 \leq p<\infty$, there exists a constant $C=C(p)>0$ such that for any $g \in \operatorname{LI}(\partial \Omega)$ and any

$$
a<C /\|g\|_{\infty}, \quad \text { we have } \quad \exp (a g) \in L^{p}(\partial \Omega) .
$$

In particular, if $\|g\|_{\infty}=0$, then $\exp (a g)$ is integrable for any $a \in \mathbb{R}$. Since for any $f \in \mathcal{D}^{\mathrm{LI}}$,

$$
\exp \left(a \mathcal{M}^{0} f\right) \in L^{p}(\partial \Omega) \Longrightarrow \exp (a f) \in L^{p}(\Omega),
$$

(6.13) also implies that for any $1 \leq p<\infty$ and $f \in \mathcal{D}^{\mathrm{LI}}$ there exists $C=C(p)>0$ such that

$$
a<C /\|f\|_{\mathcal{D}_{\infty}^{\mathrm{LI}}} \quad \Longrightarrow \quad \exp (a f) \in L^{p}(\Omega) .
$$

Definition 6.4. Let $a=a(x, u)$ be a Carathéodory function. We say that $a$ grows at most exponentially in the variable $u$, provided we can bound $a$ by

$$
a(x, u) \leq k(x) e^{A|u|}
$$

for some $A>0$ and $k \geq 0, k \in L^{s}(\Omega)$, where $s>n / 2$.

We say that $a$ grows less than exponentially in the variable $u$, provided there is $s>n / 2$ such that for any $A>0$ there is a function $k \geq 0, k \in L^{s}(\Omega)$, for which the bound (6.16) holds.

Finally, we have everything in place to proceed.

Theorem 6.5. Let $M$ be a smooth $n$-dimensional Riemannian manifold $(n \geq 3$ ) whose metric tensor $g$ is Lipschitz. Let $L$ be a strongly elliptic, (formally) selfadjoint, negative definite second-order differential operator acting on functions on $M$ that can be locally written as (3.1) with coefficients satisfying (4.17) for some $s>n$. Let $\Omega \subset M$ be a connected Lipschitz domain in $M$. Consider the following Dirichlet boundary problem:

$$
L u-a(x, u) u=f \text { in } \Omega,\left.\quad u\right|_{\partial \Omega}=g \in \operatorname{LI}(\partial \Omega), \quad u \in \mathcal{D}^{\mathrm{LI}} .
$$


Let $a(x, u) \geq 0$ be a Carathéodory function that grows at most exponentially in the variable u, i.e., (6.16) holds. Also, let $r, q$ satisfy the following conditions:

$$
r>n / 2, \quad q>\frac{n-1}{2(1-n /(2 r))} .
$$

Then there exists a constant $C>0$ such that for any given $f \in \mathcal{L}_{r}^{q}(\Omega), g \in \operatorname{LI}(\partial \Omega)$ and

$$
\|g\|_{\infty}<C / A, \quad \text { where } A \text { is the number from (6.16), }
$$

there exists a solution $u \in \mathcal{D}^{\mathrm{LI}} \cap C_{\mathrm{loc}}^{\delta}(\Omega)(\delta>0)$ to the equation (6.17). Moreover, for some $C=C(p, r)>0$

$$
\|u\|_{\mathcal{D}_{2}^{\mathrm{LI}}} \leq C\left(\|f\|_{\mathcal{L}_{r}^{q}(\Omega)}+\|g\|_{2}\right), \quad\|u\|_{\mathcal{D}_{\infty}^{\mathrm{LI}}} \leq C\|g\|_{\infty} .
$$

In particular, if the function a grows less than exponentially, the solution to (6.17) exists for all $g \in \mathrm{LI}(\partial \Omega)$.

If in addition the function $b(x, u)=a(x, u) u$ satisfies

$$
0 \leq \frac{\partial}{\partial u} b(x, u) \leq k(x) e^{A|u|},
$$

where $A$ and $k$ are the same as in Definition 6.4, then the solution $u$ to (6.17) is unique among all functions in $\mathcal{D}^{\mathrm{LI}}$ for which $\|u\|_{\mathcal{D}_{\infty}^{\mathrm{LI}}}<C / A$. In particular, if the function a grows less than exponentially, the solution $u$ is unique in $\mathcal{D}^{\mathrm{LI}}$.

Corollary 6.6. Keeping the same assumptions on $M, \Omega, L, q, r$ as in the theorem above, it follows that the Dirichlet problem

$$
L u-a(x, u) u=f \in \mathcal{L}_{r}^{q}(\Omega),\left.\quad u\right|_{\partial \Omega}=g \in \operatorname{bmo}(\partial \Omega), \quad u \in \mathcal{D}^{\mathrm{LI}},
$$

is solvable for any $g \in \operatorname{bmo}(\partial \Omega)$, provided the Carathéodory function $a(x, u) \geq 0$ grows less than exponentially in $u$.

If $a(x, u) \geq 0$ grows exponentially in $u$, then the Dirichlet problem (6.22) is solvable, provided $g$ can be written as

$$
g=g_{1}+g_{2}, \quad \text { where } g_{1} \in L^{\infty}(\Omega) \quad \& \quad\left\|g_{2}\right\|_{\mathrm{BMO}}<C / A,
$$

for some $C>0$ and $A$ from the estimate (6.16). (Said differently, (6.23) means that (6.22) is solvable for small BMO perturbations of $L^{\infty}$ boundary data g.)

Proof. We will be brief, since the main idea of the proof is the same as in Theorem 4.3. We begin with the observation we made in [3], that for fixed $r, q$ the constant $C>0$ in the estimate (3.7) can be taken independent of $2 \leq p \leq \infty$; that is, the solution $u$ to (3.6) satisfies

$$
\|u\|_{\mathcal{D}^{0, p}} \leq C\left(\|f\|_{\mathcal{L}_{r}^{q}(\Omega)}+\|g\|_{L^{p}(\partial \Omega)}\right) \quad \text { for any } 2 \leq p<\infty \text { and } g \in L^{p}(\partial \Omega) .
$$

This essentially follows from the fact that (6.24) is a result of interpolation between the estimates for $p=2$ and $p=\infty$.

Next, let the meaning of $f^{+}, f^{-}, g^{+}, g^{-}, u_{V}^{(+)}$and $u_{V}^{(-)}$be the same as in (3.14)(3.17). It follows from (3.17) that the solution $u=u_{V}=u_{V}^{(+)}-u_{V}^{(-)}$to (3.6) satisfies

$$
\left|u_{V}(x)\right| \leq u_{V}^{(+)}(x)+u_{V}^{(-)}(x) \leq h(x)=u_{0}^{(+)}(x)+u_{0}^{(-)}(x),
$$


where $u_{0}^{(+)}$and $u_{0}^{(-)}$are solutions for $V=0$ to (3.14), (3.15), respectively. Clearly (6.24) implies that

$$
\|h\|_{\mathcal{D}^{0, p}} \leq C\left(\|f\|_{\mathcal{L}_{r}^{q}(\Omega)}+\|g\|_{L^{p}(\partial \Omega)}\right) \quad \text { for any } 2 \leq p<\infty .
$$

Now, since $g \in \mathrm{LI}(\partial \Omega)$, i.e., $\|g\|_{L^{p}(\partial \Omega)} \leq C p,(6.26)$ gives us

$$
\|h\|_{\mathcal{D}_{2}^{\mathrm{LI}}} \leq C\left(\|f\|_{\mathcal{L}_{r}^{q}(\Omega)}+\|g\|_{2}\right), \quad\|h\|_{\mathcal{D}_{\infty}^{\mathrm{LI}}} \leq C\|g\|_{\infty} .
$$

Hence by (6.15) and (6.16) there exists $C>0$ such that for any $g \in \operatorname{LI}(\partial \Omega)$ satisfying $\|g\|_{\infty}<C / A$, where $A$ is the number from (6.16), we get that $V=$ $k \exp (A h) \in L^{s^{\prime}}(\Omega)$ for some $s^{\prime}>n / 2$.

It follows that the map $T: u \mapsto v$ defined by (4.27) is well defined as a map from the set

$$
\mathcal{S}=\left\{u: \Omega \rightarrow \mathbb{R}: u \in C_{\mathrm{loc}}(\Omega) \&|u(x)| \leq h(x) \text { for all } x \in \Omega \mid\right\}
$$

into itself. Notice also that the set $\mathcal{S}$ is closed and convex in the topology of $\mathcal{D}^{0, p}$ for any $2 \leq p<\infty$. As before, the map $T: \mathcal{S} \rightarrow \mathcal{S}$ is continuous and compact in the $\mathcal{D}^{0, p}$ topology; hence by the Schauder fixed point theorem, $T$ has a fixed point in $\mathcal{S}$. This proves the existence.

For uniqueness, the argument given in the proof of Theorem 4.3 goes through, provided the solutions $u_{1}, u_{2}$ that we consider satisfy

$$
\frac{\partial}{\partial u} b\left(., u_{i}(.)\right) \in L^{s^{\prime}}(\Omega), \quad \text { for some } s^{\prime}>n / 2, \quad i=1,2 .
$$

Given this and (6.21), it follows that $u_{1}=u_{2}$. (6.21) also gives that (6.29) certainly holds, provided $\left\|u_{i}\right\|_{\mathcal{D}_{\infty}^{\mathrm{LI}}} \leq C / A$.

Now we return to the discussion of Example 5.11 of 3 in the light of Theorems 5.1 and 6.5 .

Example 6.7. In [3] we considered the following situation. Let $\Omega \subset M$ be a connected Lipschitz domain on a two-dimensional compact Riemannian manifold $M$. We want to impose a given Gaussian curvature $K(x) \leq 0$ on the set $\Omega$ by conformally altering a given metric $g$ whose Gauss curvature is $k(x)$. By a well-known formula, whose proof can be found in appendix C of [23], if $g, g^{\prime}$ are conformally related, i.e., $g^{\prime}=e^{2 u} g$, then $K$ and $k$ are related by the PDE

$$
K(x)=e^{-2 u}(-\Delta u+k(x)),
$$

where $\Delta$ is the Laplace operator for the original metric tensor $g$.

As explained in [3], the fact that this problem is only two dimensional and we mainly considered the case of $\operatorname{dim} M \geq 3$ is not too important. The only reason we did not consider the case $\operatorname{dim} M=2$ in close detail is that in this case the kernel $E(x, y)$ of the inverse $L^{-1}$ of the operator (2.17) does not satisfy (2.9) but contains a logarithmic singularity of the form $\log |x-y|$. Hence it would require additional space to develop the results of section 2 for this case. However, it can be shown that all results of sections 2 and 3 (and hence also section 4 ) extend to this case. It is therefore legitimate to consider (6.30).

It follows from (6.30) that we want to solve the PDE

$$
\Delta u+K(x) e^{2 u}=k(x) .
$$


We also want to impose Dirichlet boundary conditions on $u$, i.e., we specify $g^{\prime}$ on $\partial \Omega$. Rewriting (6.31) in the form considered in Theorems 5.1 and 6.5 , we see that

$$
a(x, u)=-K(x) \frac{e^{2 u}-1}{u} \text { and } f(x)=k(x)-K(x) .
$$

Clearly, $a(x, u) \geq 0$, since $K(x) \leq 0$. It follows that (6.16) holds provided $K(x) \in$ $L^{s}(\Omega)$, for some $s>1$. If we also assume that $k \in L^{s}(\Omega)$, then all assumptions of Theorem 6.4 and Corollary 6.5 are satisfied. Notice that the uniqueness condition also holds, since $b(x, u)=-K(x)\left(e^{2 u}-1\right)$, and therefore $\frac{\partial}{\partial u} b(x, u)=-2 K(x) e^{2 u} \geq$ 0 .

It follows that there is a constant $C>0$ such that, given any $h=\left.u\right|_{\partial \Omega} \in \operatorname{LI}(\partial \Omega)$ with $\|h\|_{\infty}<C$ (or given any $h=h_{1}+h_{2}$ with $h_{1} \in L^{\infty}(\partial \Omega)$ and $\left\|h_{2}\right\|_{\mathrm{BMO}}<C$ ), we can construct a unique conformal metric $g^{\prime}$ on $\Omega$ with prescribed curvature $K(x) \leq 0, K(x) \in L^{s}(\Omega)(s>1)$ and boundary "values"

$$
\left.g^{\prime}\right|_{\partial \Omega}=\left.e^{2 h} g\right|_{\partial \Omega},
$$

where $g$ is the original metric tensor on $M$.

In some cases Theorem 5.1 also applies. It is enough to assume $K(x) \in L^{s}(\Omega)$ for some $s \geq 2$. The reason we do not want just $s>1$ is that in order to have (3.26) we need $p>s(n-1) /(s-1) n$, where $n=\operatorname{dim} M$. This condition does not represent a problem for $n \geq 3$. For $n=2$ it means that we should take $s>(1-1 / 2 p)^{-1}$, where $p$ is the regularity of the boundary data. To be on the safe side, $s \geq 2$ would always suffice.

We also need $h=\left.u\right|_{\partial \Omega} \geq 0$ and $k(x)-K(x) \leq 0$. Hence, given $k, K \in L^{s}(\Omega)$ for $s \geq 2$ such that

$$
k(x) \leq K(x) \leq 0, \quad \text { for all } x \in \Omega,
$$

then for any $h \in L^{p}(\partial \Omega), h \geq 0$, and $p>2-\varepsilon$ there is a unique conformal metric tensor $g^{\prime}$ on $\Omega$ whose curvature is $K(x)$ and has boundary "values" given by (6.33).

Notice that the solution $u$ to (6.31) is nonnegative and $g^{\prime}=e^{2 u} g$; hence at every point $x \in \Omega$ we have $g^{\prime} \geq g$. This has the following consequence. If $d_{g}$ is the distance function on $\Omega$ generated by the metric tensor $g$ and $d_{g^{\prime}}$ is the distance function generated by $g^{\prime}$, then, for any two points $x, y \in \Omega$,

$$
d_{g}(x, y) \leq d_{g^{\prime}}(x, y),
$$

i.e., the distances (and therefore also the volume of $\Omega$ ) have increased.

Due to the symmetry, there is one more case to which we can apply Theorem 5.1. Namely, if $k, K \in L^{s}(\Omega)$ with $s \geq 2$ and

$$
K(x) \leq 0, \quad K(x) \leq k(x),
$$

then for any $h \in L^{p}(\partial \Omega), h \leq 0$, and $p>2-\varepsilon$ there is a unique conformal metric tensor $g^{\prime}$ on $\Omega$ whose curvature is $K(x)$ and which has boundary "values" given by (6.35).

In this case $u \leq 0$ on $\Omega$, and so for any two points $x, y \in \Omega$ we have

$$
d_{g}(x, y) \geq d_{g^{\prime}}(x, y) .
$$

For a discussion of a different boundary value problem (with $u \rightarrow \infty$ as $x \rightarrow \partial \Omega$ ) for this PDE, see the paper by Mazzeo and Taylor [17]. 


\section{ACKNOWLEDGMENTS}

I would like to thank Michael Taylor for many helpful conversations, constant support, and interest in my work.

\section{REFERENCES}

1. J. Bergh and J. Löfström, Interpolation spaces. An introduction. Grundlehren der Mathematischen Wissenschaften, no. 223, Springer-Verlag, Berlin-New York, 1976. MR 58:2349]

2. Z.-Q. Chen, R. J. Williams and Z. Zhao, On the existence of positive solutions for semilinear elliptic equations with singular lower order coefficients and Dirichlet boundary conditions, Math. Ann. 315 (1999), 735-769. MR 2001a:35061

3. M. Dindoš, Existence and uniqueness for a semilinear elliptic problem on Lipschitz domains in Riemannian manifolds, Comm. Partial Differential Equations 27 (2002), 219-281.

4. M. Dindoš, Hardy spaces and potential theory on $C^{1}$ domains in Riemannian manifolds, Preprint (2001).

5. M. Dindoš and M. Mitrea, Semilinear Poisson Problem in Sobolev-Besov spaces on Lipschitz domains, Publicacions Matemàtiques 46 (2002), 353-403.

6. B. Dahlberg, On the Poisson integral for Lipschitz and $C^{1}$ domains, Studia Math. LXVI. (1979), 13-26. MR 81g:31007

7. B. Dahlberg and C. Kenig, Hardy spaces and the Neumann problem in $L^{p}$ for Laplace's equation in Lipschitz domains, Ann. Math. 125 (1987), 437-465. MR 88d:35044

8. B. Dahlberg, C. Kenig and G. Verchota, Boundary value problems for the system of elastostatics on Lipschitz domains, Duke Math. J. 57 (1988), 795-818. MR 90d:35259

9. E. Fabes, M. Jodeit, Jr. and N. Rivère, Potential techniques for boundary value problems on $C^{1}$-domains, Acta Math. 141 (1978), 165-186. MR 80b:31006

10. E. Fabes, C. Kenig and G. Verchota, The Dirichlet problem for the Stokes system on Lipschitz domains, Duke Math. J. 57 (1988), 769-793. MR 90d:35258

11. D. Gilbarg and N. Trudinger, Elliptic partial differential equations of second order (3rd ed.), Springer-Verlag, Berlin-New York, 1998. MR 2001k:35004

12. V. Isakov and A. I. Nachman, Global uniqueness for a two dimensional semilinear elliptic inverse problem, Trans. Amer. Math. Soc. 347 (1995), 3375-3390. MR 95m:35202

13. Z. Jin, Solvability of Dirichlet problem for semilinear elliptic equations on certain domains, Pacific J. Math. 176 (1996), 117-128. MR 97m:35082

14. F. John and L. Nirenberg, On functions of bounded mean oscillation, Comm. Pure Appl. Math. 14 (1961), 415-426. MR 24:A1348

15. C. Kenig, Harmonic analysis techniques for second order elliptic boundary value problems, CBMS Regional Conference Series in Mathematics, vol. 83, American Mathematical Society, Providence, RI, 1994. MR 96a:35040

16. C. Kenig, Elliptic boundary value problems on Lipschitz domains, Beijing Lectures in Harmonic Analysis (E. Stein, ed.), Princeton University Press, Princeton, 1986, pp. 131-183. MR 88a:35066

17. R. Mazzeo and M. Taylor, Curvature and Uniformization, Israel J. Math. 130 (2002), 323346 .

18. D. Mitrea, M. Mitrea and J. Pipher, Vector potential theory on nonsmooth domains in $\mathbb{R}^{3}$ and applications to electromagnetic scattering, J. Fourier Anal. and Appl. 3 (1997), 131-192. MR 99e:31009

19. D. Mitrea, M. Mitrea and M. Taylor, Layer potentials, the Hodge Laplacian, and global boundary problems in nonsmooth Riemannian manifolds, Memoirs Amer. Math. Soc. 150 (2001), no. 713. MR 2002g:58026

20. M. Mitrea and M. Taylor, Boundary layer methods for Lipschitz domains in Riemannian manifolds, J. Funct. Anal. 163 (1999), 181-251. MR 2000b:35050]

21. M. Mitrea and M. Taylor, Potential theory on Lipschitz domains in Riemannian manifolds: $L^{p}$, Hardy and Hölder space results, Comm. Anal. Geom. 9 (2001), no. 2, 369-421. MR 2002f:31012

22. M. Mitrea and M. Taylor, Potential theory on Lipschitz domains in Riemannian manifolds: Hölder continuous metric tensors, Comm. Partial Differential Equations 25 (2000), 14871536. MR 2001h:35040 
23. M. Taylor, Partial differential equations, vols. 1-3, Springer-Verlag, New York, 1996. MR 98b:35002b: MR 98b:35003; MR 98k:35001

24. G. Verchota, Layer potentials and regularity for the Dirichlet problem for Laplace's equation in Lipschitz domains, J. Funct. Anal. 59 (1984), 472-611. MR 86e:35038

Department of Mathematics, Malott Hall, Cornell University, Ithaca, New York $14853-4201$

E-mail address: dindos@math.cornell.edu 